\title{
Multi-wavelength observations of Asteroid 2100 Ra-Shalom
}

\author{
Michael K. Shepard ${ }^{\mathrm{a}, *}$, Beth Ellen Clark ${ }^{\mathrm{b}}$, Michael C. Nolan ${ }^{\mathrm{c}}$, Lance A.M. Benner ${ }^{\mathrm{d}}$, \\ Steven J. Ostro ${ }^{d}$, Jon D. Giorgini ${ }^{\mathrm{d}}$, Faith Vilas ${ }^{\mathrm{e}, \mathrm{n}}$, Kandy Jarvis ${ }^{\mathrm{f}}$, Susan Lederer ${ }^{\mathrm{g}}$, Lucy F. Lim ${ }^{\mathrm{h}}$, \\ Tim McConnochie ${ }^{i}$, James Bell ${ }^{i}$, Jean-Luc Margot ${ }^{i}$, Andrew Rivkin ${ }^{j}$, Christopher Magri ${ }^{k}$, \\ Daniel Scheeres ${ }^{1}$, Petr Pravec ${ }^{m}$ \\ a Department of Geography and Geosciences, Bloomsburg University of Pennsylvania, 400 E. Second St., Bloomsburg, PA 17815, USA \\ b Ithaca College, 267 Ctr for Natural Sciences, Ithaca, NY 14850, USA \\ ${ }^{\mathrm{c}}$ National Astronomy and Ionosphere Center, Cornell University, Ithaca, NY 14853, USA \\ $\mathrm{d}$ Jet Propulsion Laboratory, California Institute of Technology, Pasadena, CA 91109-8099, USA \\ e NASA Johnson Space Center, Houston, TX 77058, USA \\ ${ }^{\mathrm{f}}$ Lockheed-Martin Space Operations, 2400 NASA Rd. 1, Houston, TX 77058, USA \\ g California State University at San Bernadino, San Bernadino, CA 92407, USA \\ ${ }^{\mathrm{h}}$ NASA/Goddard Space Flight Center, Greenbelt, MD 20071, USA \\ ${ }^{\mathrm{i}}$ Cornell University, 610 Space Sciences Bldg., Ithaca, NY 14853, USA \\ j Johns Hopkins University Applied Physics Laboratory, 11100 Johns Hopkins Rd., Laurel, MD 20723, USA \\ ${ }^{\mathrm{k}}$ University of Maine, Farmington, Farmington, 336 Preble Hall, ME 04938, USA \\ ${ }^{1}$ University of Michigan, 3048 FXB, Ann Arbor, MI 48109, USA \\ $\mathrm{m}$ Astronomical Institute, Ondrejov, Czech Republic \\ ${ }^{\mathrm{n}}$ MMT Observatory, PO Box 210065, University of Arizona, Tucson, AZ 85721, USA
}

Received 28 February 2007; revised 27 August 2007

Available online 2 October 2007

\begin{abstract}
We observed near-Earth asteroid (NEA) 2100 Ra-Shalom over a six-year period, obtaining rotationally resolved spectra in the visible, nearinfrared, thermal-infrared, and radar wavelengths. We find that Ra-Shalom has an effective diameter of $D_{\text {eff }}=2.3 \pm 0.2 \mathrm{~km}$, rotation period $P=19.793 \pm 0.001 \mathrm{~h}$, visual albedo $p_{\mathrm{v}}=0.13 \pm 0.03$, radar albedo $\hat{\sigma}_{\mathrm{OC}}=0.36 \pm 0.10$, and polarization ratio $\mu_{\mathrm{c}}=0.25 \pm 0.04$. We used our radar observations to generate a three-dimensional shape model which shows several structural features of interest. Based on our thermal observations, Ra-Shalom has a high thermal inertia of $\sim 10^{3} \mathrm{~J} \mathrm{~m}^{-2} \mathrm{~s}^{-0.5} \mathrm{~K}^{-1}$, consistent with a coarse or rocky surface and the inferences of others [Harris, A.W., Davies, J.K., Green, S.F., 1998. Icarus 135, 441-450; Delbo, M., Harris, A.W., Binzel, R.P., Pravec, P., Davies, J.K., 2003. Icarus 166, 116-130]. Our spectral data indicate that Ra-Shalom is a K-class asteroid and we find excellent agreement between our spectra and laboratory spectra of the CV3 meteorite Grosnaja. Our spectra show rotation-dependent variations consistent with global variations in grain size. Our radar observations show rotation-dependent variations in radar albedo consistent with global variations in the thickness of a relatively thin regolith.
\end{abstract}

(C) 2007 Elsevier Inc. All rights reserved.

Keywords: Asteroids; Asteroids, composition; Asteroids, surfaces; Radar observations

\section{Introduction}

\subsection{Background and interest}

The Aten Asteroid 2100 Ra-Shalom was discovered in 1978

* Corresponding author. Fax: +1 5703893028.

E-mail address: mshepard@bloomu.edu (M.K. Shepard). by E. Helin (Helin et al., 1978). As one of the earlier near-Earth asteroids (NEAs) discovered, it was included in several opti- 
Table 1

Physical properties of 2100 Ra-Shalom

\begin{tabular}{ll}
\hline Property & Value \\
\hline Abs. magnitude $H$ & $16.07 \pm 0.08^{\mathrm{a}}$ \\
Optical albedo $p_{\mathrm{V}}$ & $0.04,{ }^{\mathrm{b}} 0.13^{\mathrm{c}}$ \\
Class & $\mathrm{C},{ }^{\mathrm{e}} \mathrm{S} ?^{\mathrm{c}} \mathrm{Xc}^{\mathrm{d}}$ \\
Rotation period $P(\mathrm{~h})$ & $19.797 \pm 0.003^{\mathrm{a}}$ \\
& $19.7998 \pm 0.0005^{\mathrm{f}}$ \\
$D_{\text {eff }}(\mathrm{km})$ & $3.4,{ }^{\mathrm{b}} 2.5,{ }^{\mathrm{c}} 2.4-3.6^{\mathrm{g}}$ \\
$\Delta m(\mathrm{mag})$ & $0.41^{\mathrm{a}}$ \\
$\Gamma\left(\mathrm{J} \mathrm{m}^{-2} \mathrm{~s}^{-0.5} \mathrm{~K}^{-1}\right)$ & $\geqslant 1100,{ }^{\mathrm{c}} 2500^{\mathrm{h}}$ \\
\hline
\end{tabular}

Notes. $H$ is the absolute visual magnitude, class refers to the taxonomic class, $P$ is the rotation period (synodic for Pravec et al., sidereal for Kaasalainen et al.), $D_{\text {eff }}$ is the effective diameter, $\Delta m$ indicates the observed range of the lightcurve amplitude, and $\Gamma$ is the surface thermal inertia.

a Pravec et al. (1998).

b Lebofsky et al. (1979).

c Harris et al. (1998).

d Bus and Binzel (2002b)

e Binzel et al. (2004).

f Kaasalainen et al. (2004).

$\mathrm{g}$ Shepard et al. (2000) and references therein.

h Delbo et al. (2003).

cal, thermal-infrared, and radar studies (Lebofsky et al., 1979; McFadden et al., 1984; Ostro et al., 1984; Veeder et al., 1989; Harris et al., 1998; Shepard et al., 2000). Table 1 summarizes the known physical properties of Ra-Shalom from these and more recent works.

Ra-Shalom is an intriguing asteroid for a number of reasons, as follows.

\subsubsection{Large Aten}

$\mathrm{Ra}-\mathrm{Shalom}$ is one of the larger members of the Aten family and the largest of that group studied in any detail. It is also one of the more slowly rotating members. Radar observations and lightcurve analysis by Ostro et al. (1984) provided estimates of Ra-Shalom's size and synodic rotation period of $\sim 3 \mathrm{~km}$ and $19.8 \mathrm{~h}$, respectively. The size estimate was consistent with early thermal radiometry (Lebofsky et al., 1979) and more recent estimates (Harris et al., 1998; Shepard et al., 2000). Its rotation rate has subsequently been confirmed and refined by Pravec et al. (1998) to $19.797 \pm 0.003 \mathrm{~h}$ (synodic) and by Kaasalainen et al. (2004) to $19.7998 \pm 0.0005 \mathrm{~h}$ (sidereal).

\subsubsection{Shape and evidence of surface structure}

Shepard et al. (2000), analyzing radar data acquired in 1984, determined that Ra-Shalom's convex hull has an aspect ratio $<1.2$ and noted variations in the polarization ratio that suggested variable surface roughness, perhaps caused by large scale surface structures. Kaasalainen et al. (2004) generated a three-dimensional convex shape model from 20 lightcurves obtained over four apparitions and obtained aspect ratios $a / b=$ 1.2 and $b / c=1.3$, consistent with the radar results.

\subsubsection{Spectral and classification uncertainty}

Early optical work on Ra-Shalom suggested a low visual albedo leading to a C classification (Lebofsky et al., 1979 and references therein). Harris et al. (1998) speculated that Ra-
Shalom may be an S-class based upon their moderate visual albedo estimate $\left(p_{\mathrm{v}}=0.13\right)$ and details in a ultraviolet/visible (UV/VIS) spectrum acquired by McFadden et al. (1984). Based upon high-resolution UV/VIS spectral characteristics (excluding albedo), Bus and Binzel (2002a, 2002b) classified RaShalom as an Xc-type, a generally featureless red-sloped spectrum with C-class characteristics. The most recent classification by Binzel et al. (2004), using high-resolution UV/VIS and near-infrared spectra from the small main belt asteroid spectral survey (SMASS and SMASS IR, 0.4-1.5 $\mu \mathrm{m}$ ), also placed Ra-Shalom into the $\mathrm{C}$ class.

\subsubsection{High surface thermal inertia}

Early thermal-infrared observations by Lebofsky et al. (1979) with the application of the standard thermal model (STM) gave diameters for Ra-Shalom inconsistent with (i.e. too small for) its $\mathrm{C}$ classification and expected low albedo. Additional work and the application of the thermal fast rotating model (FRM) suggested that Ra-Shalom was a member of a small group of NEAs which have a high surface thermal inertia, generally interpreted to mean lacking a surface regolith (Lebofsky et al., 1979; Veeder et al., 1989; Harris et al., 1998). Recent work by Delbo et al. (2003) suggests that Ra-Shalom may have the highest thermal inertia of any observed NEA (Table 1).

\subsubsection{Detectability of the Yarkovsky effect}

$2100 \mathrm{Ra}$-Shalom is the largest of a handful of NEAs which have observations spanning a sufficient period of time to detect the Yarkovsky effect (Vokrouhlicky et al., 2005). With additional radar observations, the effect could be detected within the next decade and the mass could be estimated. Our analysis of its size and shape could then be used to estimate its bulk density.

\subsubsection{Potential spacecraft target}

Ra-Shalom was listed as a potential target for asteroid sample return missions (Sears et al., 2001). Some advantages for including Ra-Shalom among potential sample return targets include its relatively modest $\Delta V$ for spacecraft rendezvous and its relatively slow rotation rate.

\subsection{Overview of observational campaign}

In August and September 2003, we acquired rotationally resolved (a) radar observations from Arecibo Observatory; (b) visible and near-infrared spectra (VIS) from McDonald Observatory; (c) near-infrared (IR) spectra, including $3 \mu \mathrm{m}$ to look for evidence of hydration, from the Infrared Telescope Facility (IRTF) at Mauna Kea; and (d) thermal-infrared (TIR) spectra from Palomar Observatory. In this analysis, we also make use of rotationally resolved (CW) data acquired at Arecibo in 1984 (Shepard et al., 2000), CW and delay-Doppler radar imaging of Ra-Shalom acquired at Arecibo in August 2000, and additional NIR observations at the IRTF in August 2006. A summary of all observations is listed in Table 2. 
Table 2

Observational conditions

\begin{tabular}{llllllll}
\hline Date & $\begin{array}{l}\text { RA } \\
\left({ }^{\circ}\right)\end{array}$ & $\begin{array}{l}\text { Dec } \\
\left({ }^{\circ}\right)\end{array}$ & $\begin{array}{l}\text { Phase } \\
\left({ }^{\circ}\right)\end{array}$ & $\begin{array}{l}\Delta \\
(\mathrm{AU})\end{array}$ & $\begin{array}{l}R \\
(\mathrm{AU})\end{array}$ & Mag & Observations \\
\hline 26-Aug-2000 & 355 & 21 & 31 & 0.207 & 1.183 & 14.4 & Radar \\
27-Aug-2000 & 354 & 20 & 29 & 0.204 & 1.184 & 14.3 & Radar \\
28-Aug-2000 & 352 & 19 & 27 & 0.201 & 1.185 & 14.2 & Radar \\
29-Aug-2000 & 351 & 17 & 25 & 0.199 & 1.187 & 14.1 & Radar \\
30-Aug-2000 & 350 & 16 & 23 & 0.197 & 1.188 & 14.0 & Radar \\
& & & & & & & \\
16-Aug-2003 & 314 & 41 & 48 & 0.175 & 1.121 & 14.4 & NIR \\
18-Aug-2003 & 312 & 37 & 45 & 0.174 & 1.128 & 14.3 & NIR \\
22-Aug-2003 & 308 & 29 & 40 & 0.177 & 1.141 & 14.2 & Radar, VIS, TIR \\
23-Aug-2003 & 307 & 27 & 39 & 0.179 & 1.144 & 14.2 & Radar, TIR \\
24-Aug-2003 & 306 & 24 & 38 & 0.180 & 1.147 & 14.2 & Radar, VIS \\
25-Aug-2003 & 305 & 22 & 37 & 0.182 & 1.150 & 14.2 & Radar, VIS \\
26-Aug-2003 & 305 & 20 & 36 & 0.186 & 1.153 & 14.2 & Radar \\
& & & & & & & \\
13-Aug-2006 & 248 & 34 & 74 & 0.218 & 1.050 & 15.5 & NIR \\
14-Aug-2006 & 249 & 32 & 73 & 0.219 & 1.055 & 15.5 & NIR \\
15-Aug-2006 & 251 & 30 & 72 & 0.221 & 1.060 & 15.5 & NIR \\
\hline
\end{tabular}

Notes. All parameters are listed for 00:00 UT on date listed. RA and Dec are right ascension and declination; phase is the Sun-asteroid-Earth angle (or phase angle); $\Delta$ is the asteroid-Earth distance in AU; $R$ is the heliocentric distance in AU; mag is the apparent optical magnitude; observations indicates the types of observations acquired on each date. Radar observations made at Arecibo Observatory, NIR at the IRTF, VIS at McDonald Observatory, and TIR at Palomar Observatory.

The rationale for this extensive observational campaign was to: (1) characterize the surface properties of Ra-Shalom using complementary wavelengths over a complete rotation and (2) achieve a level of surface characterization available for few NEAs. Using the radar data as a foundation, we generate a three-dimensional shape model and tie all of our spectral observations into an integrated model of surface properties.

In the next section, we briefly review the major equations, models, and conventions used in this investigation. Then we discuss the observations and reduction of optical and infrared spectroscopy, thermal-infrared spectroscopy, and radar observations. Finally, we synthesize all of these observations into a physical model of Ra-Shalom, including size, shape, composition, and rotationally resolved features.

\subsection{Background models, equations, and conventions}

In this section, we summarize the optical, thermal, and radar model parameters, equations, and uncertainty conventions that we use throughout this paper.

\subsubsection{Optical models and equations}

To place constraints on a target's diameter, we use the relationship between visual albedo $\left(p_{\mathrm{v}}\right)$, effective diameter ( $D_{\text {eff }}$, in $\mathrm{km}$ ) and absolute magnitude $(H)$ derived by Pravec and Harris (2007):

$\log p_{\mathrm{v}}=6.247-2 \log D_{\text {eff }}-0.4 H$.

The effective diameter $D_{\text {eff }}$ is the diameter of a sphere with the same projected area as the asteroid.

\subsubsection{Thermal models}

The standard thermal model (STM) assumes either a nonrotating spherical target or a rotating target with a zero surface thermal inertia (i.e., a thick regolith); in both cases, there is no nightside emission. The model includes an empirical 'beaming parameter,' $\eta$, to account for the observed enhancement of sunward emission which is thought to be a function of surface roughness and thermal inertia (Lebofsky et al., 1986). Using occultation diameters of Ceres and Pallas, Lebofsky et al. (1986) derived an empirical value of $\eta=0.756$ which is assumed to be constant for all main-belt asteroids. Lebofsky et al. (1979) developed the fast rotation model (FRM) because STM visual albedo estimates for several near-Earth asteroids were found to be significantly higher than expected from their spectral classification (see also Veeder et al., 1989). The FRM differs from the STM in that it assumes rapid rotation and no thermal beaming, or a surface of high thermal inertia (i.e., rocky with no substantial regolith; see Lebofsky and Spencer, 1989). More recently, the Near Earth Asteroid Thermal Model (NEATM) was developed by Harris (1998) to deal with the higher phase angles commonly observed in the NEA population. The NEATM differs from the STM in that the beaming parameter, $\eta$, is allowed to float to enable the model thermal continuum to be matched to that observed (Harris, 1998; Harris and Lagerros, 2002).

\subsubsection{Radar analysis}

Our observational, reduction, and analysis techniques are similar to those described by Mitchell et al. (1996), Ostro et al. (2002), and Magri et al. (2007a). Each observing cycle or "run" consisted of transmission of a circularly polarized, $2380 \mathrm{MHz}(12.6 \mathrm{~cm})$ signal for the round-trip light travel time to the target, followed by reception of echoes for the same duration in the opposite (OC) and same (SC) senses of polarization as transmitted. We operated in three modes: continuous wave (CW), delay-Doppler imaging (DD), and ranging (RNG, a coarse delay-Doppler imaging specifically used to improve ephemerides). Our reduction of raw echo power spectra included background removal, calibration, and the formation of sums of spectra weighted by signal strength.

The circular polarization ratio, $\mu_{\mathrm{c}}$, is defined to be the $\mathrm{SC} / \mathrm{OC}$ echo power ratio. It is a measure of wavelength-scale near-surface roughness. Smooth surfaces have polarization ratios approaching 0.0 , while some extremely rough surfaces have values near unity (Ostro et al., 2002). The presence of a regolith can increase $\mu_{\mathrm{c}}$ if multiple sub-surface reflections contribute significantly to the backscattering.

The OC radar albedo, $\hat{\sigma}_{\mathrm{OC}}$, of an asteroid is defined to be the ratio of its $\mathrm{OC}$ radar cross section $\left(\sigma_{\mathrm{OC}}\right)$ to its cross-sectional area,

$\hat{\sigma}_{\mathrm{OC}}=\frac{4 \sigma_{\mathrm{OC}}}{\pi D_{\mathrm{eff}}^{2}}$.

$\hat{\sigma}_{\mathrm{OC}}$ can vary with rotation and aspect. Published asteroid radar albedos vary from a low of 0.039 for the CP-class main-belt Asteroid (MBA) 247 Eukrate (Magri et al., 2007a) to a maximum 
of 0.6 for M-class 216 Kleopatra (Ostro et al., 2000). For a summary of asteroid radar properties, see http://echo.jpl.nasa.gov/ حlance/asteroid_radar_properties.html.

The instantaneous bandwidth $B$ of a radar echo is related to the radar wavelength $\lambda$ and the target's size and rotation vector by

$B(\phi)=\frac{4 \pi D(\phi)}{\lambda P} \cos \delta$,

where $D(\phi)$ is the asteroid's plane-of-sky extent normal to the apparent spin vector at rotation phase $\phi, P$ is the apparent (synodic) rotation period, and $\delta$ is the sub-radar latitude. With $D$ in $\mathrm{km}, B$ in $\mathrm{Hz}, P$ in h, and $\lambda$ of $12.6 \mathrm{~cm}$, Eq. (2) can be rewritten

$D(\phi)=\frac{P B(\phi)}{27.7 \cos \delta}$.

We estimate the minimum bandwidth and the corresponding lower bound on the maximum pole-on breadth, $D_{\max }$, using Eq. (3). Our experience with other asteroids suggests that a reasonable estimate of the bandwidth can be made from the points where the echo power spectrum first drops to two standard deviations of the background noise (referred to as two-sigma crossing thresholds).

Uncertainties in our estimates of radar cross-section (obtained by integrating the spectra) are $\pm 25 \%$ and are based on estimates of systematic uncertainties in pointing and calibration. Because systematic uncertainties are nearly the same for both polarization senses, our uncertainties in polarization ratio (ratio of SC to OC radar cross-section) are dominated by receiver noise. Unless otherwise stated, our quoted uncertainties for random errors are one-standard deviation.

\subsubsection{Rotation reference}

All of our observations are referenced to the sub-observer longitude and latitude of our radar-derived shape model (Section 3.1) at epoch 22 August 2003 00:00 UT. In this righthanded system, $0^{\circ}$ longitude (lon) is along the major axis of the model ( $+x$-axis), the $+y$-axis is perpendicular to this and intersects the equator at lon $90^{\circ}$, and the $z$-axis is perpendicular to the $x$ - and $y$-axes and parallel to the spin vector.

\section{Observations and reductions}

\subsection{Optical spectroscopy}

\subsubsection{Visible spectroscopy}

We acquired fifteen narrowband visible spectra (0.47$0.94 \mu \mathrm{m}$ ) of Ra-Shalom on 22, 24, and 25 August 2003 (UT) at the McDonald Observatory $82^{\prime \prime}$ telescope using the es 2 spectrometer coupled with a TI CCD detector. The data reduction procedures followed those described in Vilas and Smith (1985). While no clouds were apparent during the observations, humidity varied significantly throughout the nights resulting in incomplete removal of telluric water signatures from some reflectance spectra. Because the asteroid was located in a busy field near the Milky Way, exposures were occasionally paused

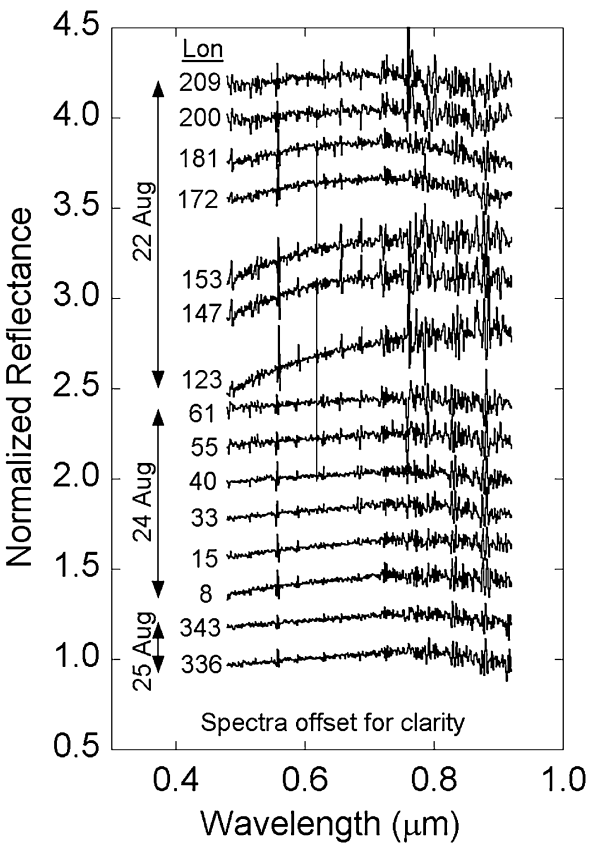

Fig. 1. Visible/near-infrared spectra acquired at McDonald Observatory in 2003. Each spectrum is normalized to a reflectance of 1.0 at $0.55 \mu \mathrm{m}$ and offset for clarity. Uncertainties are not shown but are on the order of the scatter seen in the spectra. Dates are shown on left. The sub-observer longitude at the time of acquisition, referenced to our shape model, is listed for each spectrum.

Table 3

Visible and near-infrared observations at McDonald Observatory

\begin{tabular}{llll}
\hline Date (UT) & Time (UT) & Lon $\left(^{\circ}\right)$ & AM \\
\hline 22-Aug-2003 & $04: 27$ & 209 & 1.03 \\
22-Aug-2003 & $04: 57$ & 200 & 1.01 \\
22-Aug-2003 & $06: 02$ & 181 & 1.01 \\
22-Aug-2003 & $06: 30$ & 172 & 1.03 \\
22-Aug-2003 & $07: 32$ & 153 & 1.13 \\
22-Aug-2003 & $07: 54$ & 147 & 1.19 \\
22-Aug-2003 & $09: 14$ & 123 & 1.60 \\
24-Aug-2003 & $04: 17$ & 61 & 1.03 \\
24-Aug-2003 & $04: 43$ & 55 & 1.01 \\
24-Aug-2003 & $05: 27$ & 40 & 1.01 \\
24-Aug-2003 & $05: 51$ & 33 & 1.02 \\
24-Aug-2003 & $06: 48$ & 15 & 1.12 \\
24-Aug-2003 & $07: 12$ & 8 & 1.13 \\
25-Aug-2003 & $04: 25$ & 343 & 1.02 \\
25-Aug-2003 & $04: 48$ & 336 & 1.01 \\
\hline
\end{tabular}

Notes. Time refers to the exposure start time. Lon is sub-Earth longitude of RaShalom at the midpoint of the exposure referenced to our shape model, epoch 22-Aug-2003 00:00:00 UT (JD 2452873.500). AM is airmass. Solar analog stars were SAO 126133 for exposures on 22 August 2003 and 16 Cyg B for those on 24 and 25 August 2003. Exposure integration times were $1200 \mathrm{~s}$ for all except 24 August 04:43 which was $600 \mathrm{~s}$.

(the shutter was closed) to avoid contamination when the asteroid passed close to a field star. Coupled with readout times, the total time interval encompassed by the observations comprising one spectrum can be longer than the total exposure time. Our fifteen spectra span rotation phases $46^{\circ}-284^{\circ}$ and are shown in Fig. 1. Observation circumstances are detailed in Table 3. 
Table 4

Near-infrared observations at the IRTF

\begin{tabular}{llll}
\hline UT date & UT time & Lon $\left(^{\circ}\right)$ & AM \\
\hline 16-Aug-2003 & $06: 36: 17$ & 268 & 1.39 \\
16-Aug-2003 & $07: 52: 30$ & 245 & 1.15 \\
16-Aug-2003 & $09: 28: 20$ & 216 & 1.07 \\
16-Aug-2003 & $12: 05: 09$ & 168 & 1.30 \\
16-Aug-2003* & $10: 30: 00$ & 197 & 1.07 \\
18-Aug-2003 & $06: 08: 02$ & 125 & 1.37 \\
18-Aug-2003 & $08: 49: 29$ & 76 & 1.05 \\
18-Aug-2003 & $10: 42: 10$ & 42 & 1.10 \\
18-Aug-2003 & $12: 28: 21$ & 10 & 1.37 \\
18-Aug-2003 & $13: 57: 34$ & 349 & 2.10 \\
13-Aug-2006 & & 123 & 1.03 \\
13-Aug-2006 & $05: 42: 32$ & 91 & 1.16 \\
13-Aug-2006 & $07: 30: 25$ & 68 & 1.43 \\
14-Aug-2006 & $08: 46: 28$ & 30 & 1.07 \\
14-Aug-2006 & $06: 43: 30$ & 5 & 1.25 \\
14-Aug-2006 & $08: 08: 05$ & 347 & 1.50 \\
14-Aug-2006 & $09: 05: 25$ & 339 & 1.72 \\
15-Aug-2006 & $09: 31: 42$ & 290 & 1.22 \\
\hline
\end{tabular}

Notes. Date and time are midpoints of the observations in UT. Lon is sub-Earth longitude of Ra-Shalom referenced to our shape model, epoch 22-Aug-2003 00:00:00 UT (JD 2452873.500). Airmass is in equivalent atmospheres. All spectra except the asterisked are sums of five spectra $(0.82-2.49 \mu \mathrm{m})$, each of $120 \mathrm{~s}$ integration time. The asterisked spectrum on 16-Aug-2003 is the sum of 72 spectra (LXD mode, 2.9-3.8 $\mu \mathrm{m}$ ), each of $20 \mathrm{~s}$ integration time. Standard stars for 2003 observations included Landolt 107-685, 112-1333, and 115-271. For the LXD mode spectrum, we used 16 Cyg B. For 2006, we used standard stars Landolt 107-684 and 110-361, and SAO 085632 and 085952.

\subsubsection{Infrared spectroscopy}

We acquired nine infrared (IR) spectra $(0.82-2.49 \mu \mathrm{m})$ on 2003 August 16 and 18 and an additional eight on $2006 \mathrm{Au}-$ gust $13-15$ at the $3.0 \mathrm{~m}$ NASA Infrared Telescope Facility using the 'SpeX' InSb array $(1024 \times 1024)$ spectrograph (Rayner et al., 2003). Observation circumstances are detailed in Table 4. Following normal data reduction procedures of flat fielding, sky subtraction, spectrum extraction and wavelength calibration, each spectrum was fit with the ATRAN model for telluric absorption features (Lord, 1992). Following this, each asteroid spectrum was divided by the atmospheric model at the appropriate airmass and then divided by each solar analog star spectrum, similarly reduced, and normalized at $1.0 \mu \mathrm{m}$. We obtained multiple asteroid/star ratios, minimizing chances of spurious measurements. The final spectra, shown in Fig. 2, are each averages of five contiguous spectra.

\subsubsection{3- $\mu m$ spectra}

We acquired one 3- $\mu \mathrm{m}$ spectrum on 2003 August 16 at the IRTF using the SpeX instrument in LXD grating mode (2.9$3.8 \mu \mathrm{m}$ ) (Fig. 3, Table 4). The data were reduced using Spextool, a set of IDL routines provided by the IRTF (Cushing et al., 2004). These routines include flat-fielding, wavelength calibration, image combination, and spectral extraction. A total of four data frames with an effective integration time of $320 \mathrm{~s}$ were combined for the Ra-Shalom spectrum. The solar-type star $16 \mathrm{Cyg} \mathrm{B}$ was used as a standard.

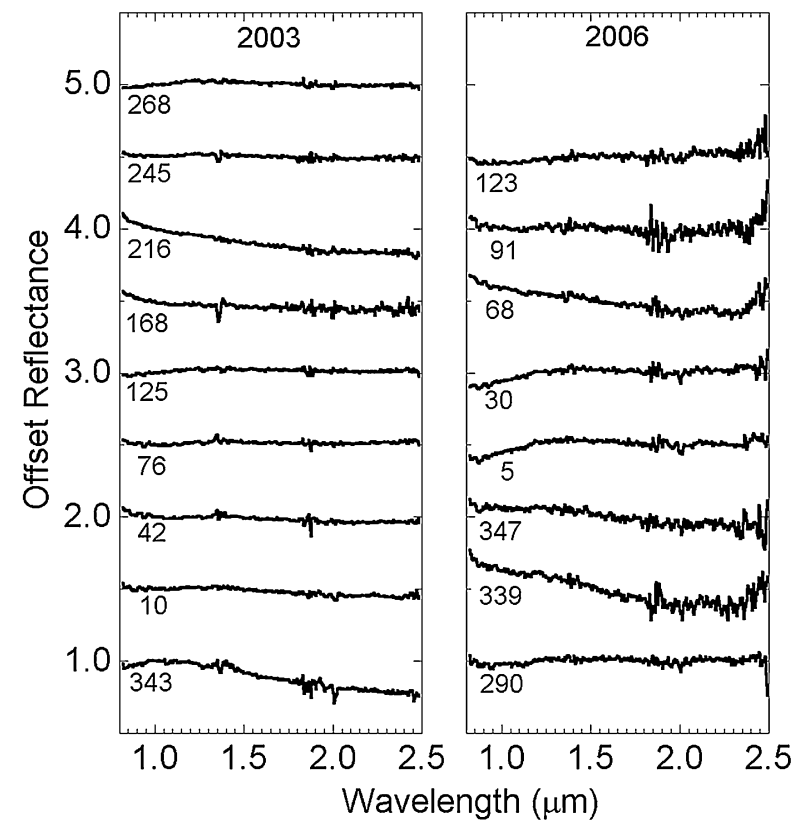

Fig. 2. IRTF spectra from 2003 (left) and 2006 (right). Each spectrum is normalized to 1.0 at $1 \mu \mathrm{m}$ and offset by 0.5 from the previous spectrum for clarity. Uncertainties are not shown but are on the order of the scatter seen in the spectra. The number under each spectrum is the sub-observer longitude at the time of acquisition (see Table 4 for dates and times), referenced to our shape model.

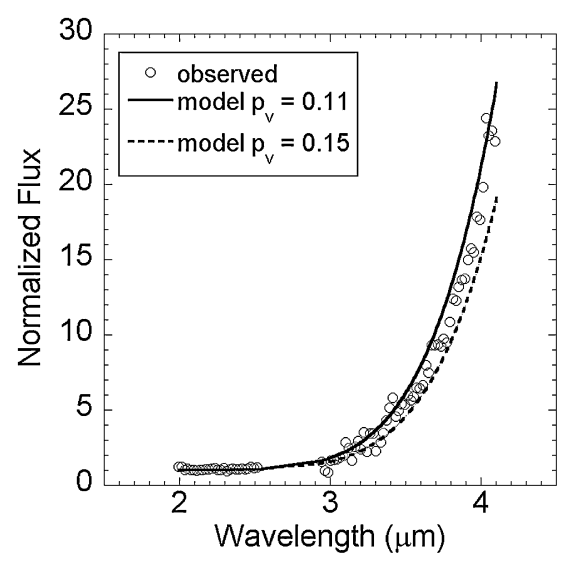

Fig. 3. IRTF LXD mode $(3 \mu \mathrm{m})$ spectra. Models assume the NEATM with $\eta=1.4$. Normalized flux is the sum of the reflected and thermal flux divided by the reflected flux so that a value of 1 is expected at shorter wavelengths where there is little or no thermal component. The asteroid spectrum has been normalized to 1.0 at $2 \mu \mathrm{m}$ for comparison.

\subsection{Thermal radiometry and spectroscopy}

Five sets of thermal-infrared spectra $(8-13.5 \mu \mathrm{m})$ of RaShalom were acquired on 2003 August 22-23 at Palomar Observatory using the Spectrocam-10 on the $200^{\prime \prime}$ Hale telescope. Observational circumstances are given in Table 5. Each spectral set is the sum of between two and four successive 160-240 s integrations (Fig. 4). In addition to low-resolution mode spectra, two camera-mode images of the asteroid were taken, both at $10.3 \mu \mathrm{m}$. Spectra were reduced according to the methods described in Lim et al. (2005a, 2005b). 
Table 5

Thermal-infrared observations at Palomar Observatory

\begin{tabular}{llll}
\hline Date (UT) & Time (UT) & Lon $\left(^{\circ}\right)$ & AM \\
\hline 22-Aug-2003 & $04: 10$ & 218 & 1.07 \\
22-Aug-2003 & $06: 58$ & 167 & 1.71 \\
23-Aug-2003 & $03: 41$ & 151 & 1.06 \\
23-Aug-2003 & $05: 33$ & 117 & 1.25 \\
23-Aug-2003 & $07: 37$ & 79 & 2.22 \\
\hline
\end{tabular}

Notes. Date and time are midpoints of the observations in UT. Lon is sub-Earth longitude of Ra-Shalom referenced to our shape model, epoch 22-Aug-2003 00:00:00 UT (JD 2452873.500). Airmass is in equivalent atmospheres. Standard stars were $\alpha$ Lyr and $\beta$ Peg.

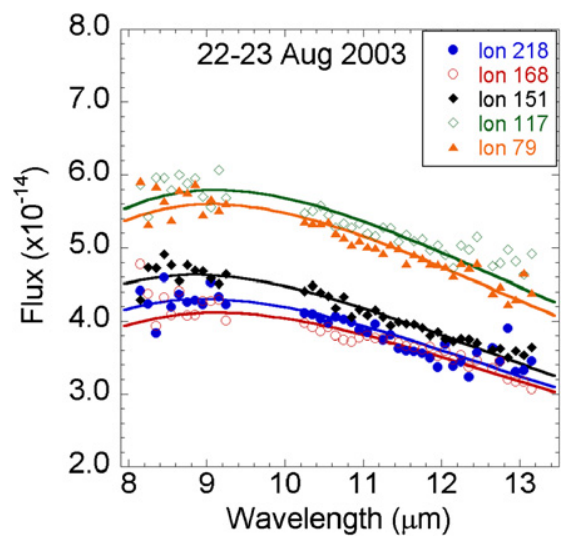

Fig. 4. Thermal IR spectra acquired at Palomar on 22-23 August 2003. Data are points, lines are standard thermal model with $\eta$ allowed to vary. Formal uncertainties are comparable to the scatter in the data. The sub-observer longitudes (referenced to our shape model) at which each data set was taken are noted in the legend (see Table 5 for dates and times). Variations in flux are consistent with changes in projected area as viewed from Earth.

\subsection{Radar observations and astrometry}

We observed Ra-Shalom on a total of ten days; $2000 \mathrm{Au}-$ gust 24-29, and 2003 August 22-26. Continuous wave observations were obtained on each day for calibration purposes (Fig. 5), and delay-Doppler imaging consumed the remaining observation time (Fig. 6). Table 6 lists the observation circumstances for both data sets.

Table 7 summarizes the $\mathrm{CW}$ observations and resultant radar properties of Ra-Shalom. The total SNR for optimally weighted and filtered CW data was 137 for the 2000 encounter and 545 for the 2003 encounter. We measured total radar cross-sections of $1.2 \pm 0.3 \mathrm{~km}^{2}$ during the 2000 encounter and $1.5 \pm 0.4 \mathrm{~km}^{2}$ during the 2003 encounter. These are consistent within their $25 \%$ absolute calibration uncertainties and with the cross-section of $1.13 \pm 0.4 \mathrm{~km}^{2}$ reported by Shepard et al. (2000) for the 1984 encounter. Circular polarization ratios (SC/OC) were consistent between the later two encounters, $0.24 \pm 0.01$ (in 2000) and $0.25 \pm 0.01$ (in 2003), but are slightly lower than the value $0.31 \pm 0.02$ reported by Shepard et al. (2000).

Corrections to Ra-Shalom's ephemeris were made after both the 2000 and 2003 radar encounters. Our final correction to the ephemeris resulted from a precise location of the asteroid center-of-mass (COM) based on our shape model (Section 3.1).
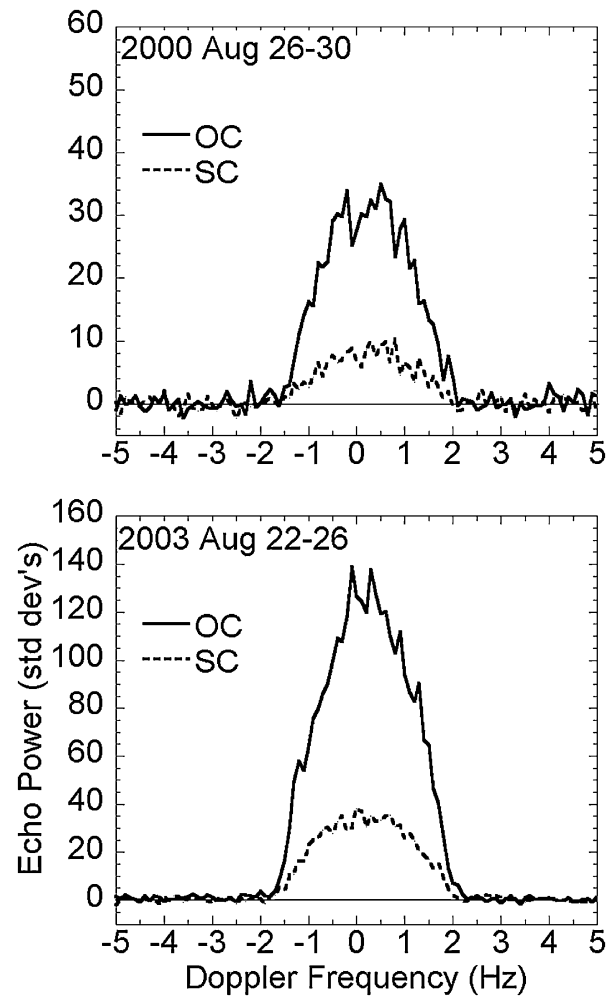

Fig. 5. Daily sums of continuous wave (CW) radar observations in 2000 and 2003 .

A correction of $15 \mu$ s was added to our original 2003 ephemeris at epoch 2003 August 22 02:15 UTC (Solution 17) which was used to generate the current best ephemeris, Solution 97 (Table 8).

\section{Analysis, synthesis, and discussion}

\subsection{Size and shape}

\subsubsection{Size constraints from thermal models}

We have two independent sets of thermal-infrared data from which we can estimate visual albedo and size. Using the 8$13 \mu \mathrm{m}$ thermal data and a thermo-physical model (Emery et al., 1998), Lim et al. (2005b) derived an estimate of Ra-Shalom's size, $D_{\text {eff }}=2.3 \pm 0.1 \mathrm{~km}$, beaming parameter, $\eta=1.2$, and placed a lower limit on its thermal inertia (assuming a perfectly smooth surface) of $580 \mathrm{~J} \mathrm{~m}^{-2} \mathrm{~s}^{-0.5} \mathrm{~K}^{-1}$. Our subsequent revision of that work leads to a slightly higher beaming parameter of $\eta=1$.4. If the surface is rough, the thermal inertia increases accordingly. This value is at least an order of magnitude larger than a typical lunar regolith $\left(\sim 50 \mathrm{~J} \mathrm{~m}^{-2} \mathrm{~s}^{-0.5} \mathrm{~K}^{-1}\right)$, comparable to values reported by Harris et al. (1998) and Delbo et al. (2003) (Table 1), and suggests a near-surface (centimeter depth scale) dominated by coarse or coherent rock-either bedrock or boulders larger than a few wavelengths. Using Eq. (1), the absolute magnitude (Table 1) and this diameter leads to a visual albedo $p_{\mathrm{v}}=0.13$.

Our estimate of $\eta$ is comparable to the value, $\eta=1.3$, computed using our solar phase angle and the mean behavior of NEAs (Delbo et al., 2003), but slightly lower than values re- 


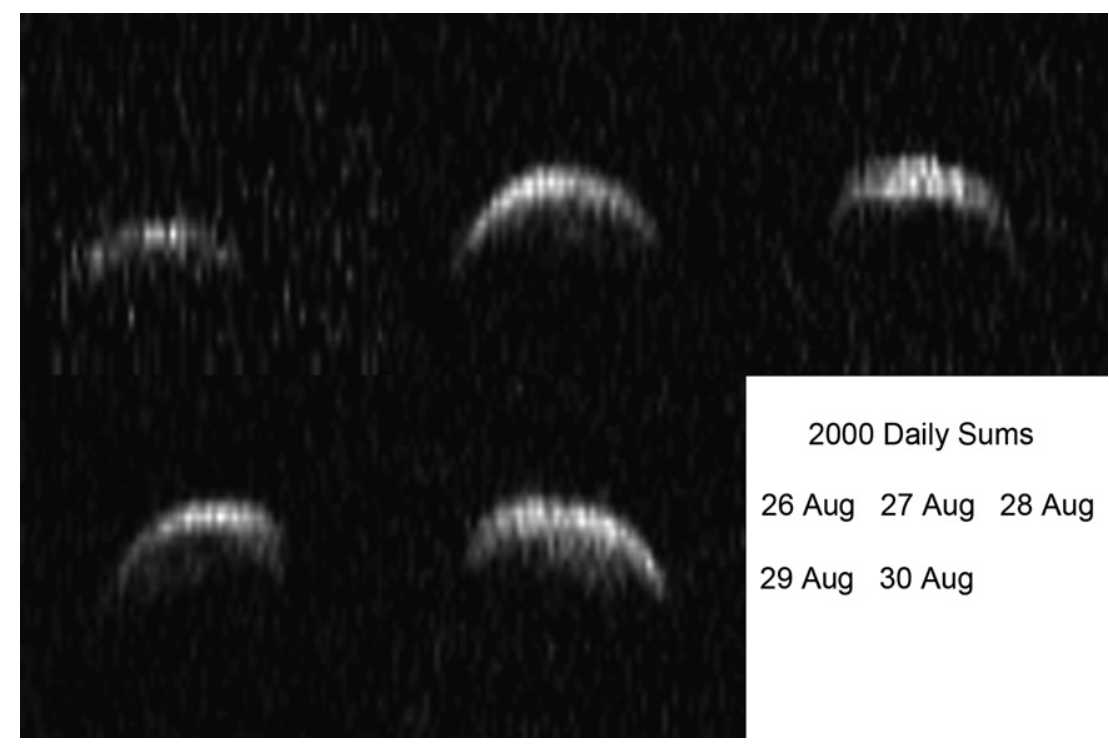

(a)

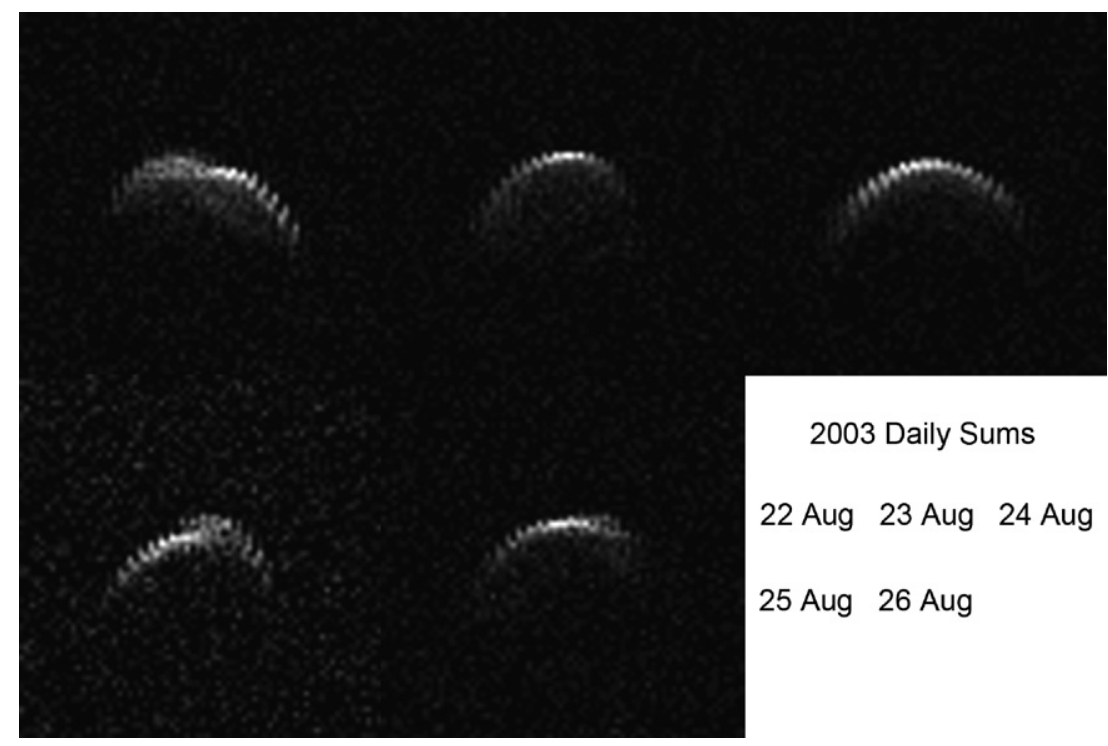

(b)

Fig. 6. Mosaic of radar delay-Doppler images acquired in 2000 (a) and 2003 (b). Each image is the sum of all images taken that day. Delay increases from top to bottom, Doppler frequency from left to right. Image resolution is scaled to $75 \mathrm{~m} /$ pixel in range, $0.1 \mathrm{~Hz}$ in Doppler. Each image is $10 \mathrm{~Hz}$ wide and $7.5 \mathrm{~km}$ in delay.

ported by Harris et al. (1998) $(\eta=1.6-1.8)$ and Delbo et al. (2003) $(\eta=2.3)$. However, direct comparisons of $\eta$ with previous work may not be strictly valid because of differences in viewing aspect, solar phase angle, or surface inhomogeneities.

Next, we fit our $3 \mu \mathrm{m}$ IRTF data (LXD mode) using the NEATM model. Using $\eta=1.4$, we find that albedos of $p_{\mathrm{v}}=$ $0.13 \pm 0.02$ give the best fits, corresponding to $D_{\text {eff }}=2.3 \pm$ $0.2 \mathrm{~km}$, and consistent with the thermal data (Fig. 3). The consistency between our thermal and $3 \mu \mathrm{m}$ data leads us to consider $D_{\text {eff }}=2.3 \pm 0.2 \mathrm{~km}$ and $p_{\mathrm{v}}=0.13 \pm 0.03$ as our best estimates. These values agree with previous work by Harris et al. (1998).

\subsubsection{Size, shape, and pole constraints from $C W$ radar data}

To place constraints on Ra-Shalom's pole, we use estimated bandwidths and positions from the 1981, 1984, 2000, and 2003 radar encounters. For the 2000 and 2003 observations, we use 2sigma threshold bandwidth estimates from the sum of all observations during an encounter, giving an estimate of the minimum $D_{\max }$ (Table 7). The SNRs for the 1981 and 1984 encounters were considerably lower. For the 1981 encounter we use the 4-8 Hz bounds set by Ostro et al. (1984), and for the 1984 encounter we use the $3.7 \pm 0.3 \mathrm{~Hz}$ bandwidth of the best fit convex hull (Shepard et al., 2000). Ra-Shalom's total movement across the sky was small during each encounter $\left(\sim 10^{\circ}\right.$ total), so we assume the position in the middle of the encounter for our analyses. For a given $D_{\max }$, we can calculate the expected bandwidth (BW) for every possible pole and compare this to our measured values. Fig. 7 shows a contour plot of $\chi^{2}$ residuals for $D_{\max }$ of $2.9 \mathrm{~km}$. The asterisks show the sky position of Ra-Shalom at the center of each encounter. The pole reported by Kaasalainen et al. (2004), derived from an inversion 
Table 6

Radar observations

\begin{tabular}{|c|c|c|c|c|}
\hline Date & $\begin{array}{l}\text { Receive } \\
\text { start-end } \\
\text { UTC h }\end{array}$ & \#Runs & Mode & Resolution \\
\hline \multirow[t]{2}{*}{ 26-Aug-2000 } & $4.50-6.17$ & 8 & $\mathrm{CW}$ & $0.1 \mathrm{~Hz}$ \\
\hline & & 5 & $\mathrm{DD}$ & $0.1 \mathrm{~Hz} / 4 \mu \mathrm{s}$ \\
\hline \multirow[t]{2}{*}{ 27-Aug-2000 } & $4.47-6.88$ & 2 & $\mathrm{CW}$ & $0.1 \mathrm{~Hz}$ \\
\hline & & 19 & DD & $0.1 \mathrm{~Hz} / 2 \mu \mathrm{s}$ \\
\hline \multirow[t]{2}{*}{ 28-Aug-2000 } & $4.19-5.71$ & 2 & $\mathrm{CW}$ & $0.1 \mathrm{~Hz}$ \\
\hline & & 10 & DD & $0.1 \mathrm{~Hz} / 2 \mu \mathrm{s}$ \\
\hline \multirow[t]{2}{*}{ 29-Aug-2000 } & $4.37-6.63$ & 2 & $\mathrm{CW}$ & $0.1 \mathrm{~Hz}$ \\
\hline & & 19 & $\mathrm{DD}$ & $0.1 \mathrm{~Hz} / 2 \mu \mathrm{s}$ \\
\hline \multirow[t]{2}{*}{ 30-Aug-2000 } & $4.23-6.42$ & 2 & $\mathrm{CW}$ & $0.1 \mathrm{~Hz}$ \\
\hline & & 18 & DD & $0.1 \mathrm{~Hz} / 2 \mu \mathrm{s}$ \\
\hline \multirow[t]{2}{*}{ 22-Aug-2003 } & $1.80-4.13$ & 5 & $\mathrm{CW}$ & $0.05 \mathrm{~Hz}$ \\
\hline & & 18 & DD & $0.1 \mathrm{~Hz} / 1 \mu \mathrm{s}$ \\
\hline \multirow[t]{2}{*}{ 23-Aug-2003 } & $1.59-4.08$ & 6 & $\mathrm{CW}$ & $0.05 \mathrm{~Hz}$ \\
\hline & & 18 & DD & $0.1 \mathrm{~Hz} / 1 \mu \mathrm{s}$ \\
\hline \multirow[t]{2}{*}{ 24-Aug-2003 } & $1.43-4.01$ & 4 & $\mathrm{CW}$ & $0.05 \mathrm{~Hz}$ \\
\hline & & 21 & DD & $0.1 \mathrm{~Hz} / 1 \mu \mathrm{s}$ \\
\hline \multirow[t]{2}{*}{ 25-Aug-2003 } & $1.29-3.87$ & 6 & $\mathrm{CW}$ & $0.05 \mathrm{~Hz}$ \\
\hline & & 12 & DD & $0.1 \mathrm{~Hz} / 1 \mu \mathrm{s}$ \\
\hline \multirow[t]{2}{*}{ 26-Aug-2003 } & $1.17-3.73$ & 3 & $\mathrm{CW}$ & $0.05 \mathrm{~Hz}$ \\
\hline & & 9 & DD & $0.1 \mathrm{~Hz} / 1 \mu \mathrm{s}$ \\
\hline
\end{tabular}

Notes. Receive time indicates the beginning of first and last reception; number of runs refers to number of transmit and receive cycles; mode indicates continuous wave (CW) or delay-Doppler (DD); resolution gives the frequency and delay resolution. In all cases, the delay resolution refers to the baud rate, while the sampling rate was half of this. For example, a $2 \mu$ s baud ( $300 \mathrm{~m}$ resolution) was sampled at $1 \mu \mathrm{s}$, giving an effective delay-depth resolution of $150 \mathrm{~m}$.

Table 7

Disk integrated radar properties from $\mathrm{CW}$ observations

\begin{tabular}{lcclll}
\hline Date & SNR & Lon $\left(^{\circ}\right)$ & $\mathrm{BW}(\mathrm{Hz})$ & $\sigma_{\mathrm{oc}}\left(\mathrm{km}^{2}\right)$ & $\mu_{\mathrm{c}}$ \\
\hline 26-Aug-2000 & 82 & 199 & $3.2 \pm 0.1$ & $1.04 \pm 0.26$ & $0.24 \pm 0.01$ \\
27-Aug-2000 & 40 & 131 & $2.3+0.9 /-0.5$ & $1.14 \pm 0.29$ & $0.33 \pm 0.02$ \\
28-Aug-2000 & 48 & 60 & $2.7+0.4 /-0.1$ & $1.35 \pm 0.34$ & $0.25 \pm 0.02$ \\
29-Aug-2000 & 58 & 340 & $3.1+0.2 /-0.1$ & $1.23 \pm 0.31$ & $0.23 \pm 0.02$ \\
30-Aug-2000 & 69 & 229 & $3.2+0.4 /-0.1$ & $1.26 \pm 0.32$ & $0.22 \pm 0.01$ \\
2000 CW total & 137 & \multicolumn{1}{l}{-} & $3.6+0.1 /-0.2$ & $1.16 \pm 0.29$ & $0.24 \pm 0.01$ \\
22-Aug-2003 & 287 & 240 & $4.0 \pm 0.1$ & $1.63 \pm 0.41$ & $0.24 \pm 0.01$ \\
23-Aug-2003 & 233 & 160 & $3.3 \pm 0.1$ & $1.23 \pm 0.31$ & $0.25 \pm 0.01$ \\
24-Aug-2003 & 195 & 92 & $4.2+0.1 /-0.3$ & $1.51 \pm 0.38$ & $0.28 \pm 0.01$ \\
25-Aug-2003 & 307 & 22 & $3.6 \pm 0.1$ & $1.53 \pm 0.38$ & $0.26 \pm 0.01$ \\
26-Aug-2003 & 176 & 306 & $3.9+0.1 /-0.3$ & $1.51 \pm 0.38$ & $0.27 \pm 0.01$ \\
2003 CW total & 545 & - & $4.0+0.3 /-0.1$ & $1.48 \pm 0.37$ & $0.25 \pm 0.01$
\end{tabular}

Notes. SNR is the total signal-to-noise for the $\mathrm{CW}$ observations on that date; Lon is the sub-radar longitude of Ra-Shalom at the midpoint of the daily CW observations, referenced to epoch 22-Aug-2003 00:00:00 UT; BW is the 2-standard-deviations-of-noise crossing threshold and the uncertainties are based on the 1- and 3-stdevs crossing threshold. $\sigma_{\mathrm{oc}}$ is the OC radar crosssection and $\mu_{\mathrm{c}}$ is the polarization ratio.

of lightcurves, is shown as a ' $\mathrm{P}$ ' within a circle and is consistent with our constraints. If we require the existence of at least one pole with $\chi^{2} \leqslant 1.0$, our joint constraints on bandwidth require $D_{\text {max }}$ to be $2.9 \pm 0.2 \mathrm{~km}$.

Using the maximum-to-minimum bandwidth ratio measured during each encounter, we estimate the aspect ratio of Ra-Shalom to be 1.4 (2000 encounter) and 1.2 (2003 en-
Table 8

Orbital elements

\begin{tabular}{lll}
\hline Quantity & Value & Uncertainty \\
\hline Eccentricity & 0.43645840209684 & $\pm 2.645 \mathrm{e}-08$ \\
Perihelion distance & 0.468902636279023 & $\pm 2.1933 \mathrm{e}-08 \mathrm{AU}$ \\
Perihelion date & 2453878.193184100734 & $\pm 1.0327 \mathrm{e}-05 \mathrm{~d}$ \\
& $(2006-$ May-22.69318411) &
\end{tabular}

Long. asc. node

Arg. perihelion

170.8768709933894

355.9952122442684

$\pm 8.4204 \mathrm{e}-06^{\circ}$

$\pm 1.0587 \mathrm{e}-05^{\circ}$

Inclination

15.7574349146213

Semimajor axis

Period

0.8320639293066

277.2256879888752

$\pm 6.1035 \mathrm{e}-06^{\circ}$

Mean anomaly

$\pm 6.1956 \mathrm{e}-10 \mathrm{AU}$

$\pm 3.0964 \mathrm{e}-07 \mathrm{~d}(0.76 \mathrm{y})$

$\pm 1.3466 \mathrm{e}-05^{\circ}$

Notes. $2100 \mathrm{Ra}$-Shalom heliocentric J2000 orbital elements (OSOD \#97). Uncertainties are one standard deviation. Epoch JD 2454000.5 = 2006 Sep 22.0.

counter). These compare favorably with the estimate of 1.2 by Shepard et al. (2000), the relatively low lightcurve amplitude of 0.4 mag (Pravec et al., 1998), and the shape model of Kaasalainen et al. (2004).

\subsubsection{Size, shape, and rotation state from radar images}

We used the shape modeling method of Hudson (1993) to generate a three-dimensional (3D) shape model from all of the imaging and $\mathrm{CW}$ data available. Details of the inversion algorithm and process are given by Ostro et al. (2005) and Magri et al. (2007b). Because we are dealing with data spanning nearly two decades, the rotation period becomes a critical parameter to refine. We did this by locating common features on radar images from the 2000 and 2003 encounters (Fig. 8). These images appear to be at approximately the same sub-radar longitude, so there must have been an integer number of revolutions in the time interval between these observations (assuming no sky motion - an approximation which is refined with the shape modeling). A brief analysis showed two possible periods, 19.792 and $19.807 \mathrm{~h}$, which bracket the published values by Pravec et al. (1998) and Kaasalainen et al. (2004). Of these two periods, the former is lower than the values reported by Pravec et al. (1998) and Kaasalainen et al. (2004), while the latter is similar to an alternate value reported by Kaasalainen et al. (2004). The period favored by Kaasalainen et al. (2004) $(19.7998 \mathrm{~h}$ ) forces our images to be a half-rotation out of phase and is excluded.

In our initial shape modeling efforts, we attempted to use both the radar data and lightcurves from encounters in 1997, 2000, and 2003. We found it difficult to fit both data sets satisfactorily; either the model radar images corresponded closely to the observed images but the lightcurves were badly fit, or the lightcurves were well fit but the model radar images were a poor match to those observed. As we discuss below (Section 3.2), we believe there is evidence for rotational variations in RaShalom's albedo which may affect lightcurves and contribute to this difficulty. For this reason, we excluded lightcurves from our shape analysis.

We ran hundreds of simulations using a variety of size, shape, and pole states to locate the model with the lowest $\chi^{2}$ residual. Our best model is shown in Figs. 9-12 and detailed in 


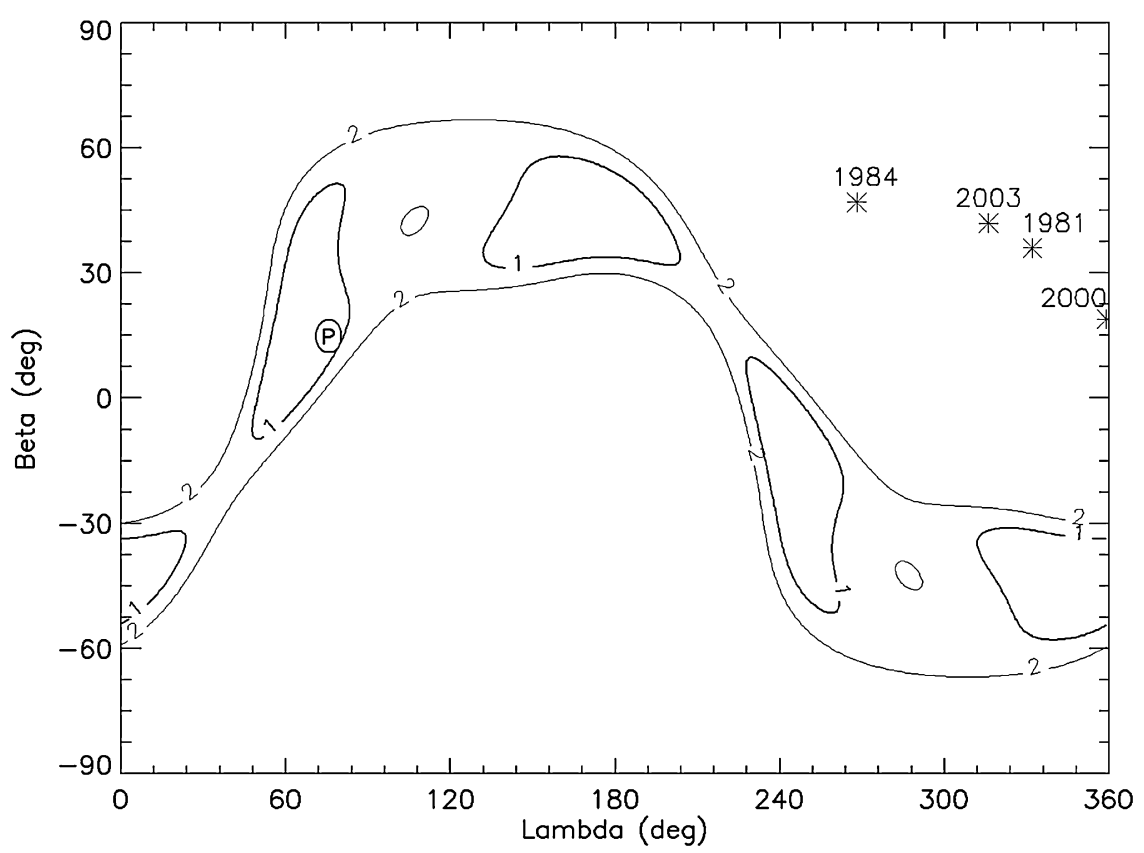

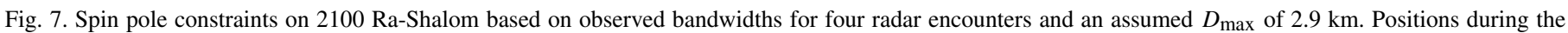

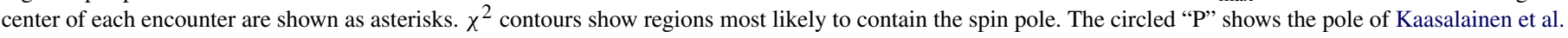
(2004) which is within $3^{\circ}$ of our model pole.

Table 9. Our model has 1148 surface elements, each with an average area of $(90 \mathrm{~m})^{2}$, a good match to our imaging resolution of 75 to $150 \mathrm{~m}$ per pixel. The model is volumetrically equivalent to an ellipsoid of dimensions $2.8 \times 2.4 \times 1.8 \mathrm{~km}$ and has an effective diameter of $2.3 \pm 0.2 \mathrm{~km}$, consistent with our infrared data and analysis and previous size estimates (Table 1). The surface area of our model is $17.5 \mathrm{~km}^{2}$, which is only $7 \%$ larger than the surface area of an equivalent sphere, indicating relatively smooth topography at the hectometer scale.

Fig. 13 shows our shape model to be consistent with the hull of Ra-Shalom previously reported (Shepard et al., 2000). Our estimate of its sidereal rotational period is $19.793 \pm 0.001 \mathrm{~h}$. To tie our shape model to IRTF observations in 2006, we attempted but failed to obtain either radar observations or a lightcurve in that year. However, the precision of our estimated rotational period results in longitude uncertainties of only $\pm 15^{\circ}$ in the three years between the epoch of our shape model and observations in 2006.

Because there is only $\sim 75^{\circ}$ total sky motion between all encounters, we are unable to constrain the pole beyond that shown in Fig. 7. Our best solutions have ecliptic longitudes $\lambda=75^{\circ} \pm 10^{\circ}$, consistent with the pole reported by Kaasalainen et al. (2004). Our constraints on ecliptic latitude are less rigorous. The solution shown and adopted has $\beta=16^{\circ}$, also consistent with Kaasalainen et al. (2004), but we found equally good shape models with latitudes up to $\beta=60^{\circ}$. We were unable to rule out retrograde rotation (mirror solutions of that given) or the alternate period of $19.808 \mathrm{~h}$. For purposes of modeling and discussion, we adopted the best model with a pole consistent with Kaasalainen et al. (2004). Our results do not significantly change if we adopt models using the other poles or rotational period.
Shape modeling involves many subjective decisions and we tried to strike a compromise between excluding features in the images that were real (penalty weights too large) and including spurious shape features caused by image noise (penalty weights too small). In this particular case, there are several obvious structural features apparent in our images and model (Fig. 11). Foremost is the large protrusion at lon $\sim 0^{\circ}$. While the two ends $\left(0^{\circ}\right.$ and $\left.180^{\circ}\right)$ are similar, the $0^{\circ}$ end is more pointed and has a definite structural feature evident in the images while the $180^{\circ}$ end is more rounded. There is also evidence of several depressions, the most obvious at $275^{\circ}$ near the southern pole.

There are two shape model features that are intriguing, but difficult to confirm by visual inspection of the individual images and we cannot rule out a spurious cause. The first of these are north/south running linear ridges near the protrusion (located near lon $\sim 30^{\circ}$ and best observed in the lon $90^{\circ}$ view of Fig. 11). These are reminiscent of structural features observed on 433 Eros (Prockter et al., 2002). The second feature is the pointed knob near the north pole (best observed in the lon $270^{\circ}$ view of Fig. 11 at the top, right center). This may be a structure near the resolution limit of our images $(\sim 100 \mathrm{~m})$, perhaps similar to the large boulders and knobs seen on Itokawa (Fujiwara et al., 2006).

\subsection{Composition}

\subsubsection{Albedo}

Given our best estimate of its size $\left(D_{\text {eff }}=2.3 \pm 0.2\right)$ and estimated absolute magnitude $(16.07 \pm 0.08)$, Ra-Shalom's visual albedo is $0.13 \pm 0.03$. This value is brighter than the traditional C-class albedo $(\leqslant 0.10)$ and consistent with the lower end of the S-class. 


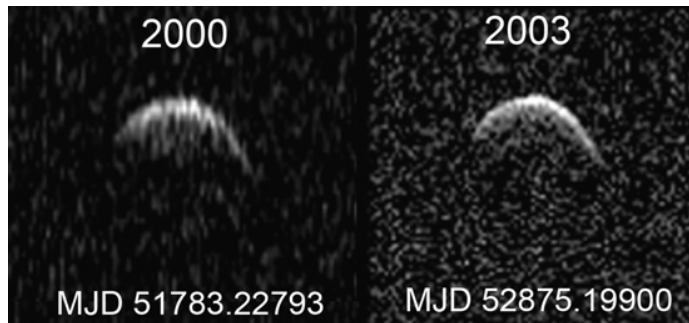

MJD 51785.21766
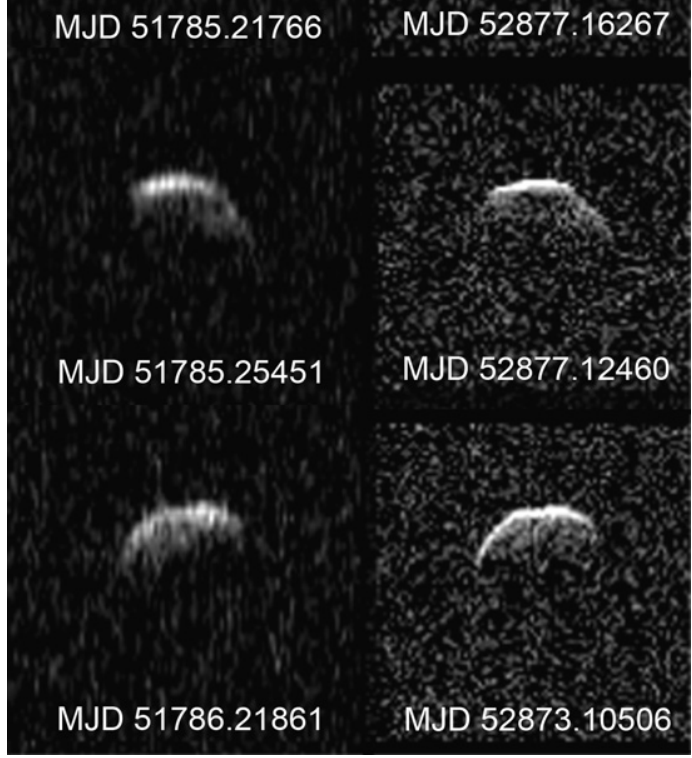

Fig. 8. Delay-Doppler images from 2000 and 2003 that appear to be at similar sub-radar longitudes and the date (modified Julian) at which each image was acquired. Each image has range increasing toward bottom, resolution $75 \mathrm{~m} / \mathrm{pixel}$, and Doppler frequency increasing left to right, $0.1 \mathrm{~Hz}$ resolution. Each image is $10 \mathrm{~Hz}$ wide and $7.5 \mathrm{~km}$ in delay.

\subsubsection{Thermal infrared spectra}

Lim et al. (2005b) removed the blackbody continuum from the thermal spectra in Fig. 4 and found no evidence of emission bands at the 5\% level.

\subsubsection{Composite spectra}

VIS and IR spectra from 2003 were combined where rotation phases were within a few degrees of one another (Fig. 14). Significant rotational variations are evident. Unfortunately, RaShalom was in the Milky Way during all our observations in 2003 making it difficult to rule out background sources. Our observations in August 2006 at the IRTF were designed to check this variability (Fig. 15). Those observations confirm the spectral variability, and despite different sub-observer latitudes $\left(-5^{\circ}\right.$ versus $\left.+20^{\circ}\right)$, solar phase angles $\left(45^{\circ}\right.$ versus $\left.72^{\circ}\right)$, and uncertainty in the sub-observer longitude in $2006\left( \pm 15^{\circ}\right)$, there is considerable, though not complete agreement at equivalent longitudes.
We conducted two checks on the fidelity of our spectra. First, we compared the sum of our 2003 and 2006 IR spectra (Fig. 16). The two data sets are in agreement except for an upturn in the 2006 data after $2.3 \mu \mathrm{m}$; this may be a thermal feature because Ra-Shalom was closer to the Sun in 2006 than 2003 (Table 2). As a second check, we used our shape model to compute the sub-observer longitude for the dates and times of the SMASS (13 September 1997 09:12 UT, lon $\sim 204^{\circ}$ ) and SMASS IR (30 September 1997 11:30 UT, lon $\sim 310^{\circ}$ ) spectra (Binzel et al., 2004; Binzel, personal communication) and compared those with our spectra closest in aspect. As is evident in Fig. 17, our results closely correspond to the SMASS results where they overlap, supporting both the rotation period and fidelity of our spectra.

Three spectral 'themes' are evident in our IRTF observations. The first is a flat spectrum, similar to those observed in C-class asteroids with evidence of broad and shallow $1 \mu \mathrm{m}$ and/or $2 \mu \mathrm{m}$ absorption features. The second is a negative or blue slope that was also observed in the SMASSIR survey (Fig. 17). The third is a spectrum with a red-slope up to $1.3 \mu \mathrm{m}$ and flat thereafter. The relatively coarse sampling in rotation phase and whole disk spectra makes it difficult to positively associate any spectral type with the structural features evident in our shape model. However, there do appear to be correlations of the IR blue-slope with high radar albedos. We discuss this further in Section 3.5.

\subsubsection{Spectral class and meteorite analog (CV3)}

To compare our spectra with those of meteorites, we averaged all of our normalized spectra (Fig. 18), including the 2003 and 2006 IR observations. Our composite spectrum is most similar to laboratory spectra of CV3 meteorites. In particular, we find an excellent agreement with the CV3 Grosnaja (Johnson and Fanale, 1973; Gaffey, 1976), especially in the infrared. ${ }^{1}$ This meteorite is primarily olivine with accessory pyroxene and a carbon coating on the grain surfaces, lowering its overall albedo (Gaffey, personal communication). Given the purported link between K-class asteroids (Eos-like) and CV3/CO3 meteorites (Bell, 1988; Burbine et al., 2001), this suggests RaShalom would be more accurately classified as a K-type. This is consistent with our estimate of Ra-Shalom's visual albedo $\left(p_{\mathrm{v}}=0.13\right)$; K-class asteroids have a mean visual albedo of $0.15 \pm 0.05$ (based on all $25 \mathrm{~K}$-class asteroids currently in the JPL Horizons database, http://ssd.jpl.nasa.gov/horizons.cgi).

Our single 3- $\mu \mathrm{m}$ IRTF spectrum (Fig. 3) does not show evidence of a water-of-hydration band depth greater than roughly $20 \%$ (or about one standard deviation of noise). This is consistent with our interpretation of a CV3 composition as these

\footnotetext{
1 We noted some inconsistencies between the laboratory measurements of Grosnaja samples published by Johnson and Fanale (1973) and Gaffey (1976). Both groups independently measured the same samples, but the published spectra show different slopes in the IR and the two larger size separates display different shapes in the VIS. We are unsure of the reasons for this and have therefore used both sets in our analysis and note which were used in the captions.
} 


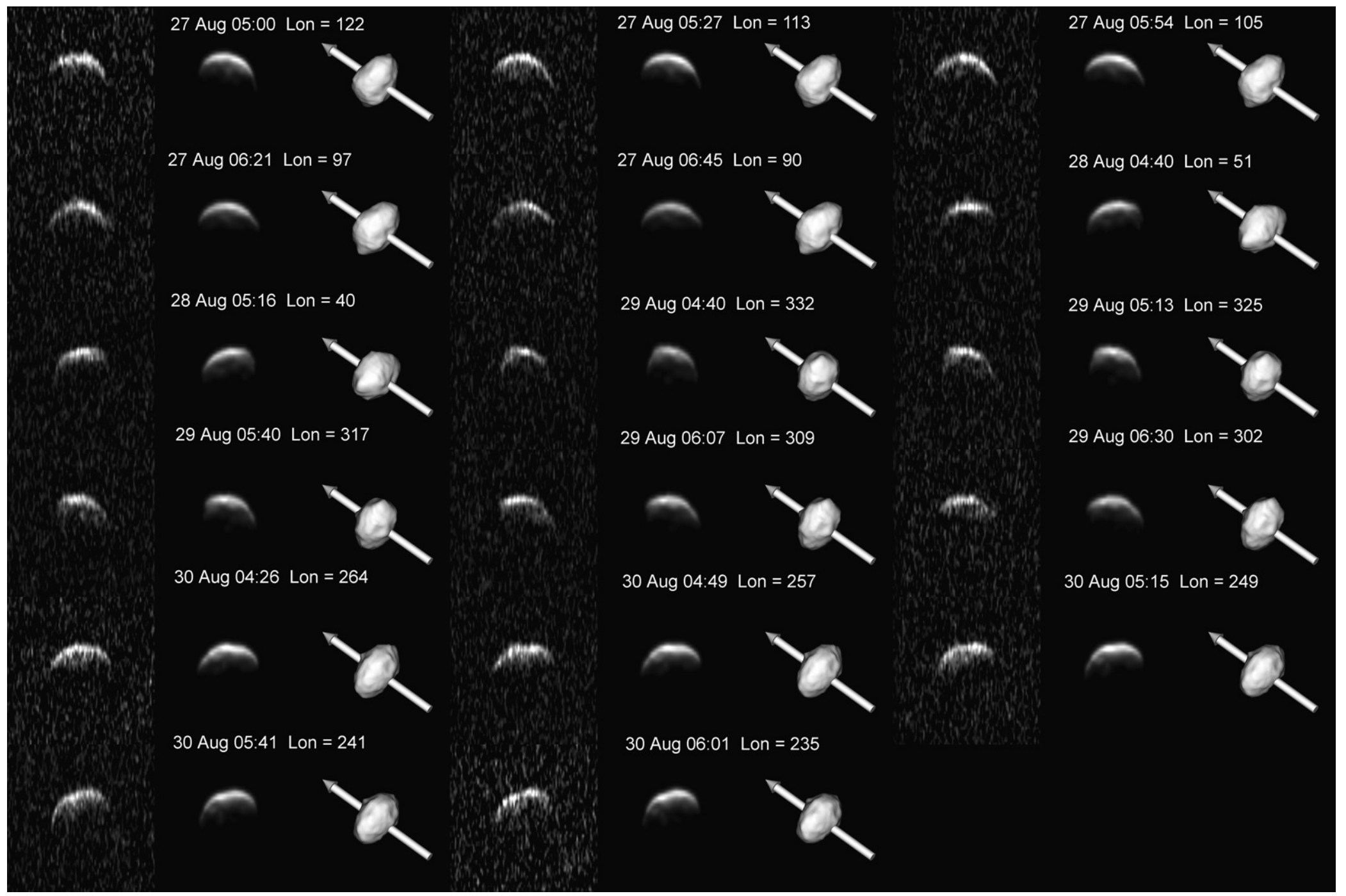

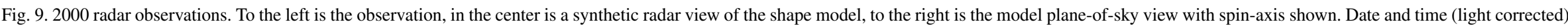
and sub-radar longitude are shown. 


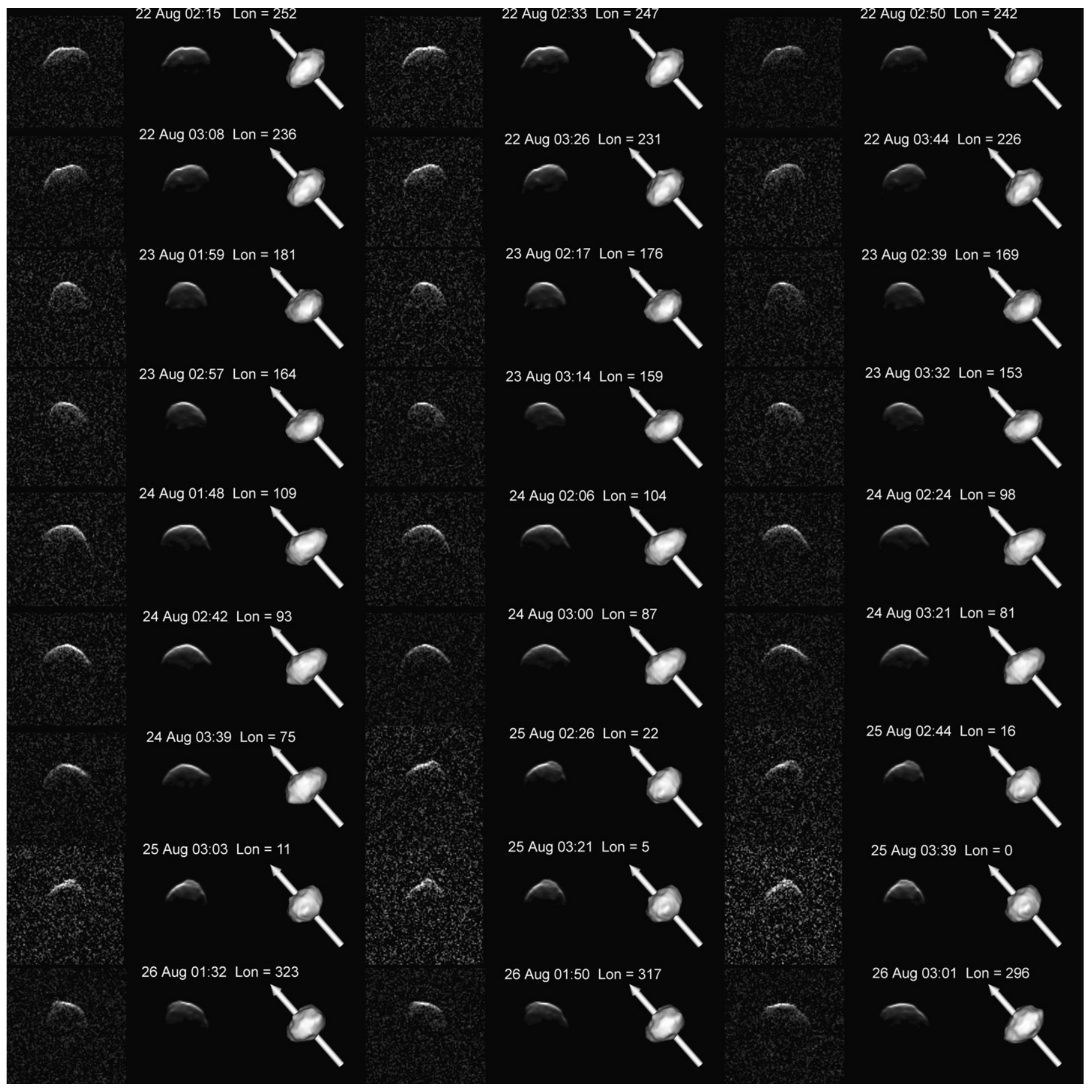

Fig. 10. 2003 radar observations. To the left is the observation, in the center is a synthetic radar view of the shape model, to the right is the model plane-of-sky view with spin-axis shown. Date and time (light corrected) and sub-radar longitude are shown.

meteorites are generally anhydrous and have weak or absent bands at $3 \mu \mathrm{m}$.

Most of our spectra do not have an IR blue slope. Those that do are very similar to the lab spectra of the larger grain size fractions (Fig. 19). The 'bluing' of the spectrum with increasing grain size is common with CO3/CV3 meteorites (Johnson and Fanale, 1973; Gaffey, personal communication). Additionally, the albedo of the fine-grain laboratory sample is $\sim 10-12 \%$ while that of the coarse fractions are $\sim 5 \%$ (Johnson and Fanale,
1973). The agreement of our average spectrum with the finegrained Grosnaja spectrum indicates that fines are prevalent over most of the surface.

It is difficult to associate specific regions with particular spectra because of the whole disc nature of our observations. However, for low to moderate solar phase angles $\left(<45^{\circ}\right)$ the regions most likely to dominate the spectra are those within a few tens of degrees of the sub-observer point. This is the case for the 2003 observations where solar phase angles were $\sim 40^{\circ}$. 


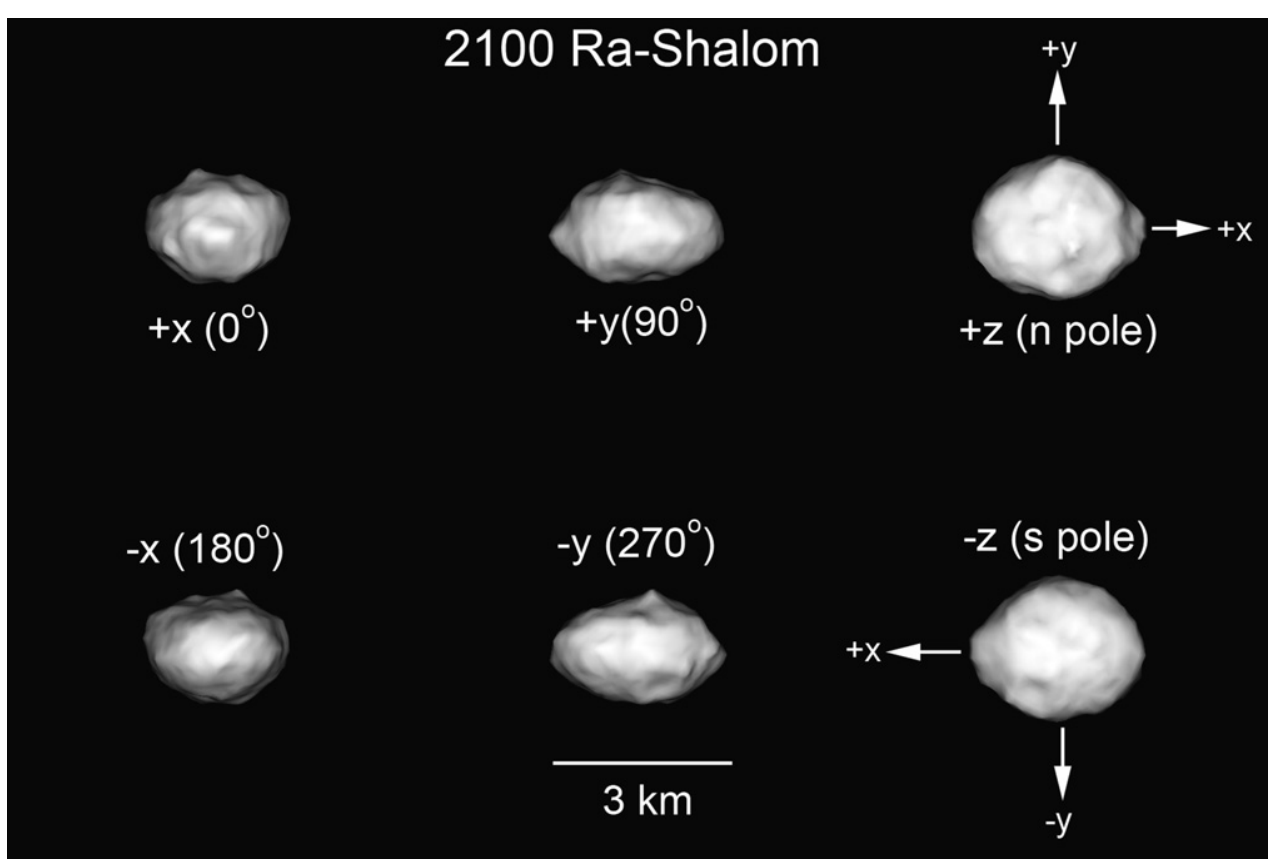

Fig. 11. Shape model of 2100 Ra-Shalom. Model details are given in Table 9. Axes and longitudes (in parentheses) refer to axis pointing toward viewer.

For higher phase angles, like those in 2006 (solar phase angles of $72^{\circ}$ ) the most important regions are those from the sub-observer longitude up to the sub-solar longitude, or approximately one "solar phase angle" (Sun-asteroid-Earth) away from the sub-observer longitude. For an observation centered on lon $0^{\circ}$, the spectra could be dominated by terrain from anywhere between lon $0^{\circ}$ and $72^{\circ}$; this wide-range makes it difficult to narrow down the part(s) of the surface contributing the most to each spectrum.

\subsection{Radar constraints on the near-surface}

The OC radar cross-section (obtained from the calibrated $\mathrm{CW}$ data) varies with longitude. For most asteroids, this type of variation is due to shape-larger projected areas at the time of an observation give rise to higher radar cross-sections. To remove this effect, we normalized the radar cross-section to the projected area of our shape model at the times of the observations (Fig. 20) and plotted the normalized radar albedo with the projected area. We find that variations in the asteroid shape and projected area are not sufficient to explain the observed changes in radar cross-section. The region centered on lon $270^{\circ}$ shows a very high radar albedo of $\sim 0.45$, a value typically associated only with $\mathrm{M}$-asteroids. A similarly high radar albedo is observed at lon $\sim 30^{\circ}$, coinciding with a low projected area. Lower radar albedos, although still quite high, of $\sim 0.34$ are observed near the respective ends (lon $0^{\circ}$ and $180^{\circ}$ ). The 2000 radar albedo data show a similar trend but are systematically offset by -0.05 from the 2003 data and are within our absolute uncertainties.

Are these radar albedos consistent with our spectral and thermal data? Although fraught with uncertainties, there are models purporting to estimate near-surface bulk density or porosity given a radar albedo, polarization ratio, and grain density. Magri et al. (2001) derive two such models; one using 433 Eros as a calibration standard (calibrated model) and the other based on radar scattering models (uncalibrated model). Assuming a grain density of $3.5 \mathrm{~g} \mathrm{~cm}^{-3}$ (mean for $\mathrm{CO} / \mathrm{CV}$ chondrites; Britt and Consolmagno, 2003), both models predict near-surface porosities of $25 \pm 15 \%$. This is considerably lower than found on the Moon (40-60\%; Carrier et al., 1991) and is consistent with bare rock.

The polarization ratio is sensitive to the roughness of the surface within a few wavelengths depth. Variations in $\mu_{\mathrm{c}}$ are therefore indicative of changes in regolith thickness, porosity, particle size distribution, and/or surface roughness at one or more interfaces. Ra-Shalom's mean polarization ratio is $0.25 \pm 0.01$ (Table 7), considerably lower than the mean of $0.35 \pm 0.24$ for NEAs (L.A.M. Benner et al., in preparation), and is consistent with a thin or regolith-free surface. With one exception, we observe only small variations in polarization ratio with rotation longitude, suggesting the near-surface (within $\sim 10-40 \mathrm{~cm}$ ) roughness is approximately homogeneous. The exception is in observations acquired at sub-radar lon $\sim 130^{\circ}$, lat $-23^{\circ}$ in the 2000 data. There are no obvious features in the shape model at this longitude (Fig. 11), but we note that this region also has a relatively low radar albedo (Fig. 20).

\subsection{Surface environment of Ra-Shalom}

Given Ra-Shalom's shape, size, and rotation state, it becomes possible to evaluate the dynamical environment on the surface of the asteroid (Scheeres et al., 1996). Based on spectral type, we assume a grain density of $3.5 \mathrm{~g} \mathrm{~cm}^{-3}$ (Britt et al., 2002; Britt and Consolmagno, 2003). Britt et al. (2002) report an average macroporosity of $30 \%$ for known C-class asteroids, consistent with our previous estimate of Ra-Shalom's surface bulk density $(25 \% \pm 15 \%$, Section 3.3$)$. Assuming this value for Ra- 

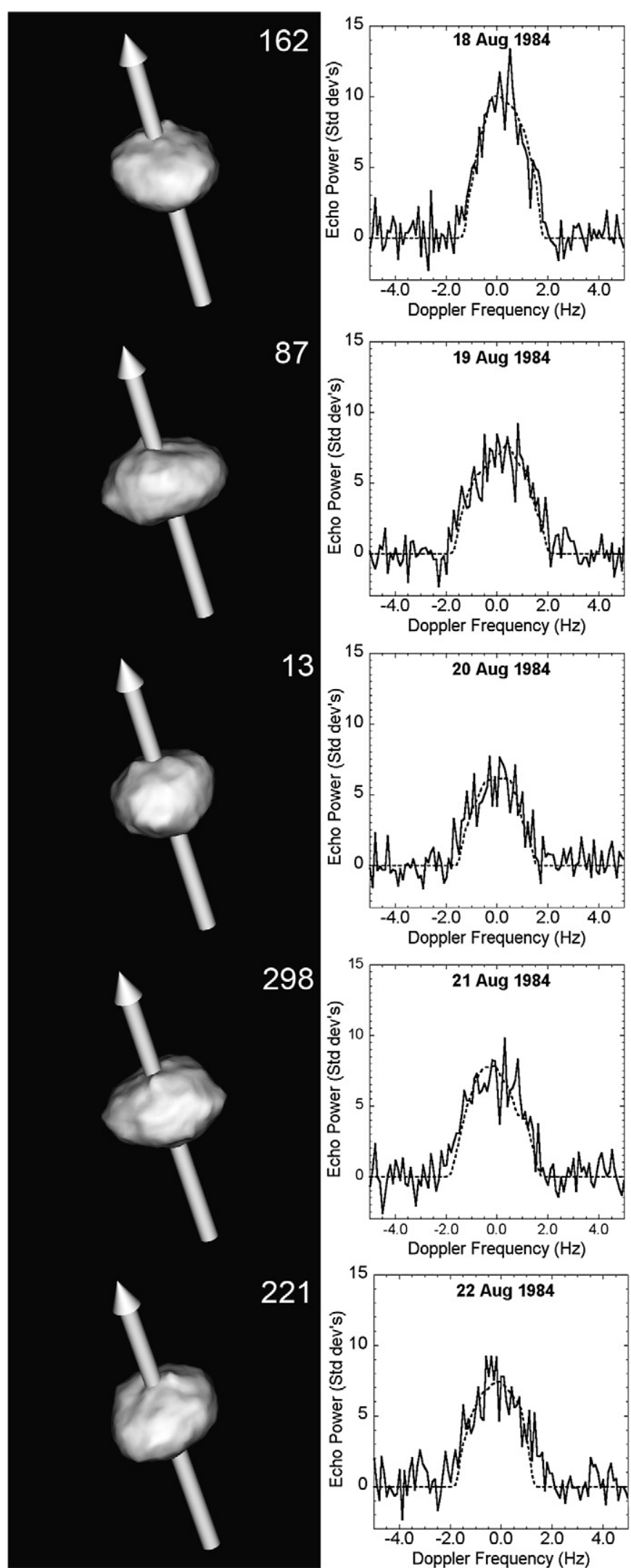

Fig. 12. Plane-of-sky views (left) and model fits (dashed) to daily sums of 1984 CW data (solid). Longitude, referenced to shape model, is in upper left corner of plane-of-sky views.
Table 9

Properties of the shape model

\begin{tabular}{ll}
\hline Maximum dimensions & \\
$\quad x$-axis & $2.96 \mathrm{~km} \pm 10 \%$ \\
$y$-axis & $2.46 \mathrm{~km} \pm 10 \%$ \\
$z$-axis & $1.95 \mathrm{~km} \pm 10 \%$ \\
Pole $(\lambda, \beta)$ & $75^{\circ} \pm 10^{\circ}, 16^{\circ} \pm 15^{\circ}$ \\
Rotation period $(\mathrm{h})$ & $19.793 \pm 0.001$ \\
Surface area $\left(\mathrm{km}^{2}\right):$ & $17.5 \pm 20 \%$ \\
Volume $\left(\mathrm{km}^{3}\right):$ & $6.2 \pm 30 \%$ \\
$D_{\text {eff }}(\mathrm{km})$ & $2.3 \pm 0.2$ \\
DEEVE $(\mathrm{km})$ & $2.80 \times 2.37 \times 1.78 \pm 10 \%$ \\
\hline
\end{tabular}

Notes. $D_{\text {eff }}$ is the diameter of a sphere with the model's volume; DEEVE is dimensions of the dynamically equivalent equal-volume ellipsoid, a homogeneous ellipsoid having the same moment-of-inertia ratios and volume as model.

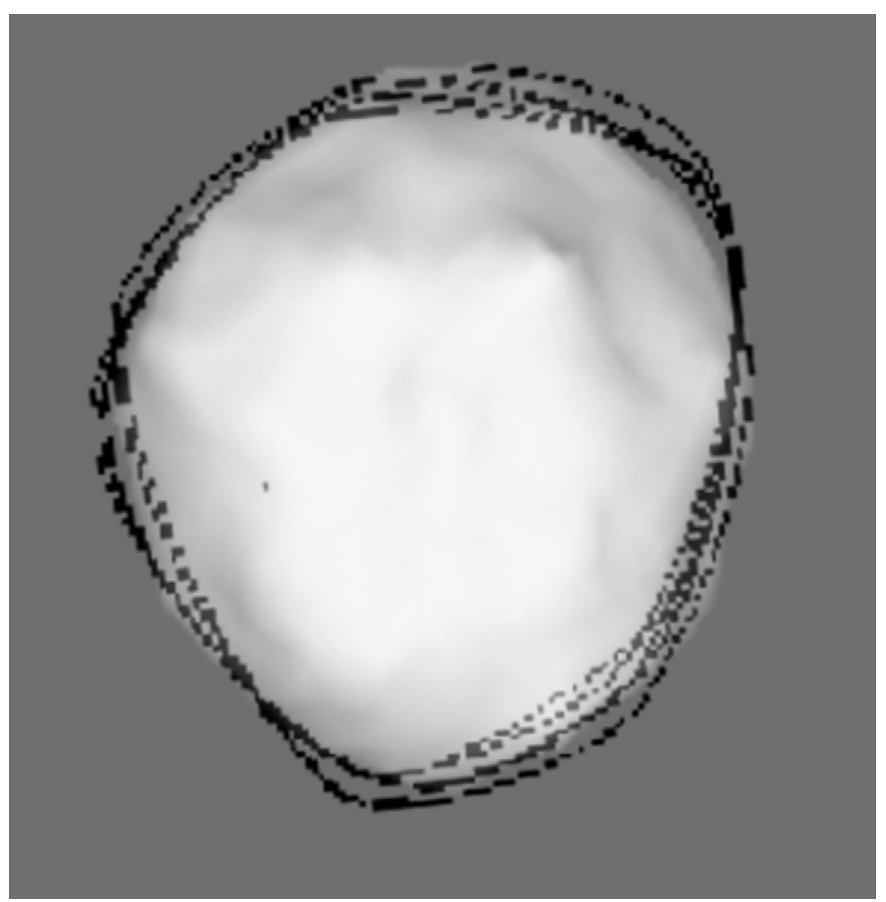

Fig. 13. Shape model as viewed from above compared with five hull models generated from $1984 \mathrm{CW}$ data (Shepard et al., 2000). The shape view is centered on (lon, lat) $\left(180^{\circ},+64^{\circ}\right)$ to simulate the hull aspect of the 1984 encounter. The hull has been rotated to coincide with the correct rotational phase before superimposing on the shape model.

Shalom leads to a bulk density estimate of $2.4 \pm 0.6 \mathrm{~g} \mathrm{~cm}^{-3}$ and escape speeds at the surface of 1.16 to $1.55 \mathrm{~m} \mathrm{~s}^{-1}$.

Over the surface of Ra-Shalom, the local acceleration ranges from 0.654 to $0.776 \mathrm{~mm} \mathrm{~s}^{-2}$. As it rotates about its maximum moment of inertia axis, particles on the surface will feel an additional centrifugal acceleration that will only range up to $1.2 \times 10^{-2} \mathrm{~mm} \mathrm{~s}^{-2}$, about $2 \%$ of its weak gravitational attraction. Thus, unlike asteroids with more rapid spin rates, the surface dynamics on Ra-Shalom are dominated by gravity alone.

Gravitational slopes average 13 degrees over the surface. We computed distributions of surface slopes and found them to exceed $10^{\circ}$ over $66 \%$ of the surface, $15^{\circ}$ over $36 \%$ of the surface, and $30^{\circ}$ over only $2 \%$ of the surface. The largest slopes are about $40^{\circ}$ and occur on the more prominent outcroppings of 

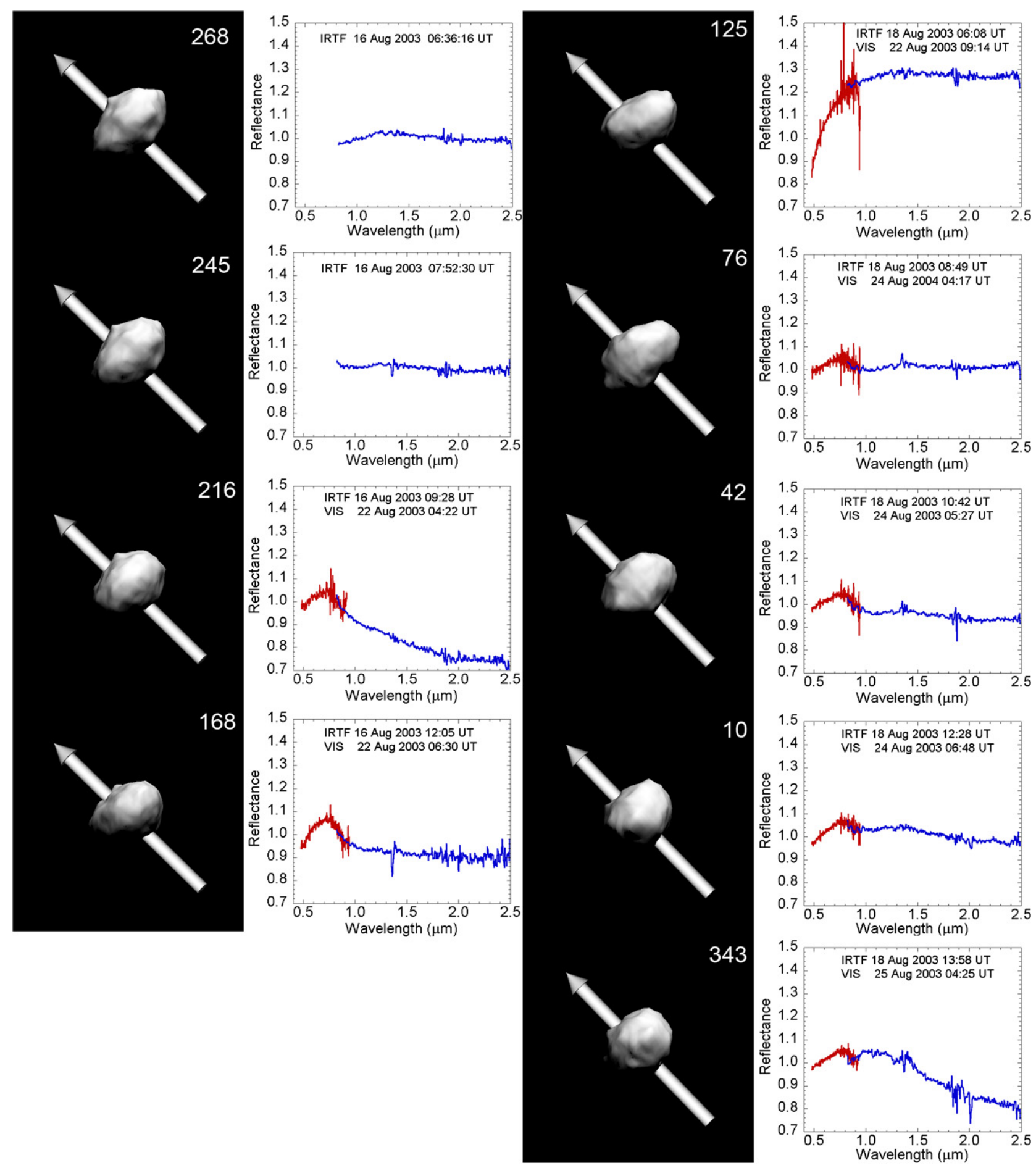

6

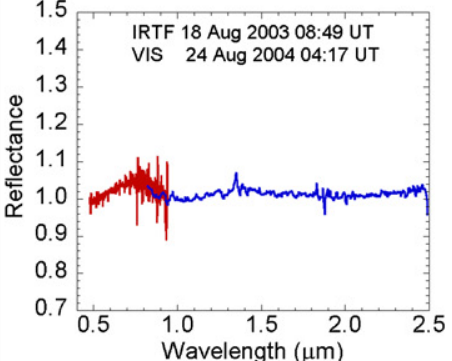

42

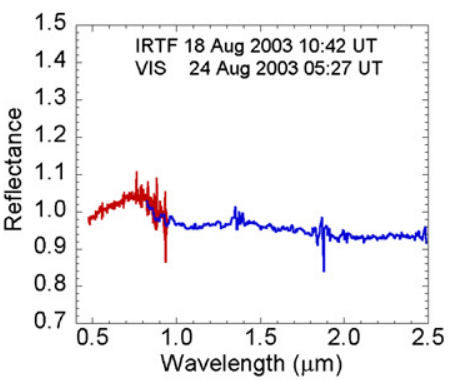

10
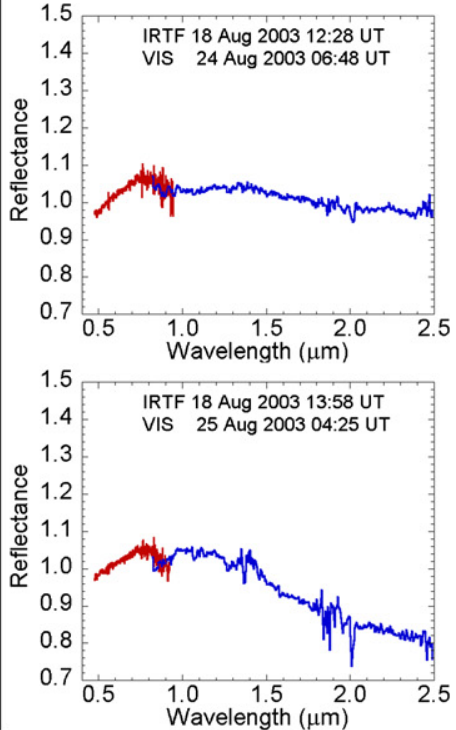

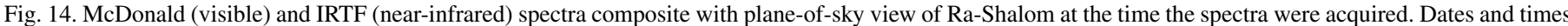

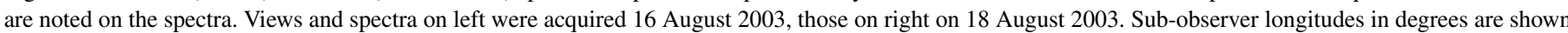

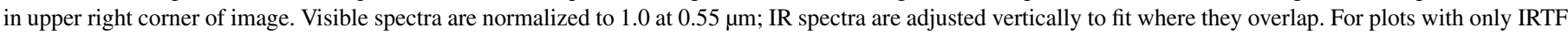
spectra, they are normalized to 1.0 at $1 \mu \mathrm{m}$.

the asteroid. Slopes at this value are at the large end of the distribution for the angle of repose for unconsolidated piles of particles. Therefore it is possible that those regions are relatively regolith-free, with some exposure of bedrock. If regolith is free to migrate, it will tend towards the rotation poles of the body, as they are the potential lows for Ra-Shalom given its relatively slow rotation rate (Guibout and Scheeres, 2003).

\subsection{Synthesis}

How can these observations from a variety of wavelengths be combined and interpreted? To aid the visualization, we have superposed the longitudes corresponding to our work and previous thermal observations (Harris et al., 1998; Delbo et al., 2003) and IR spectra onto Fig. 20. We categorized our IR spec- 

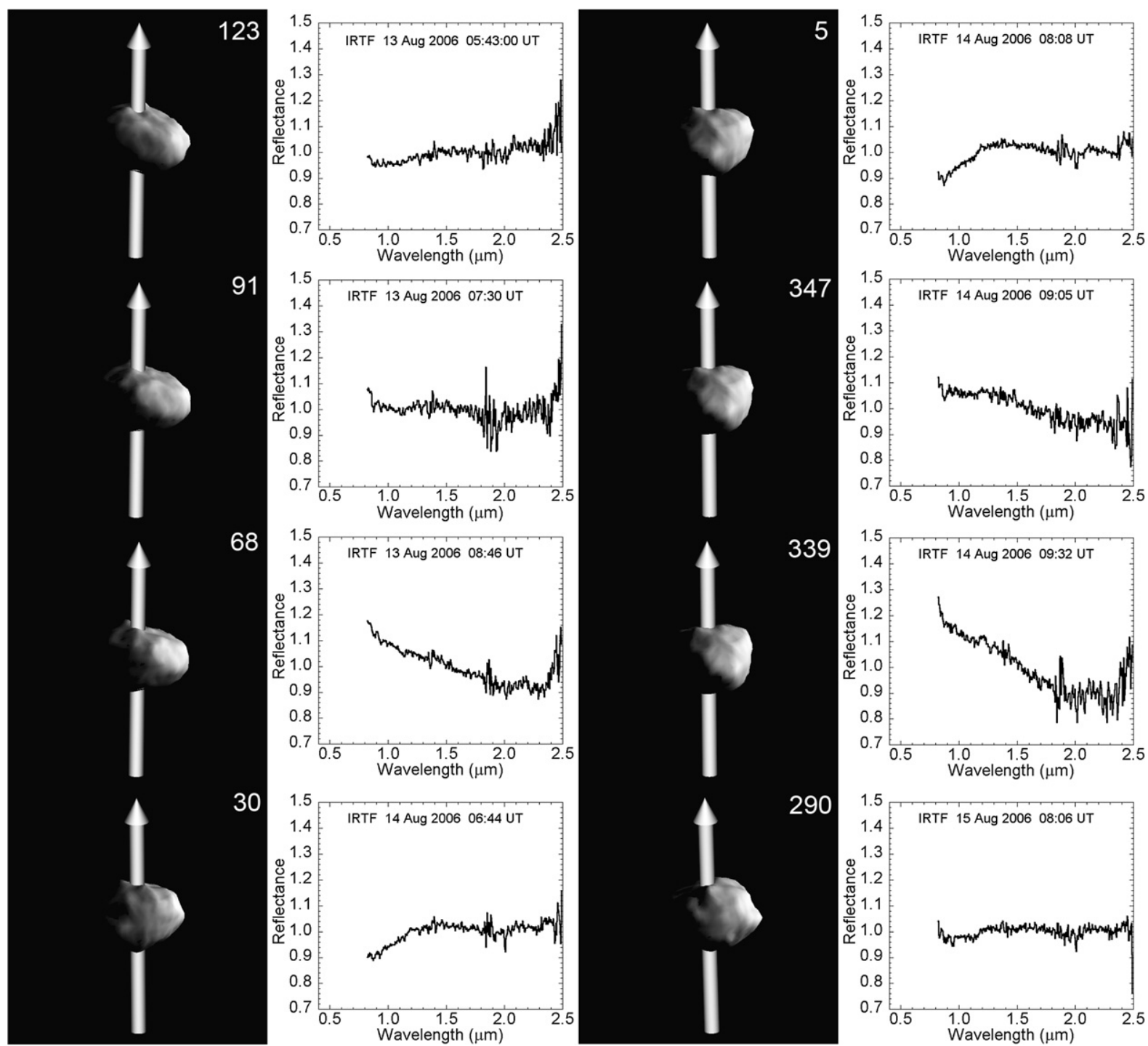

347
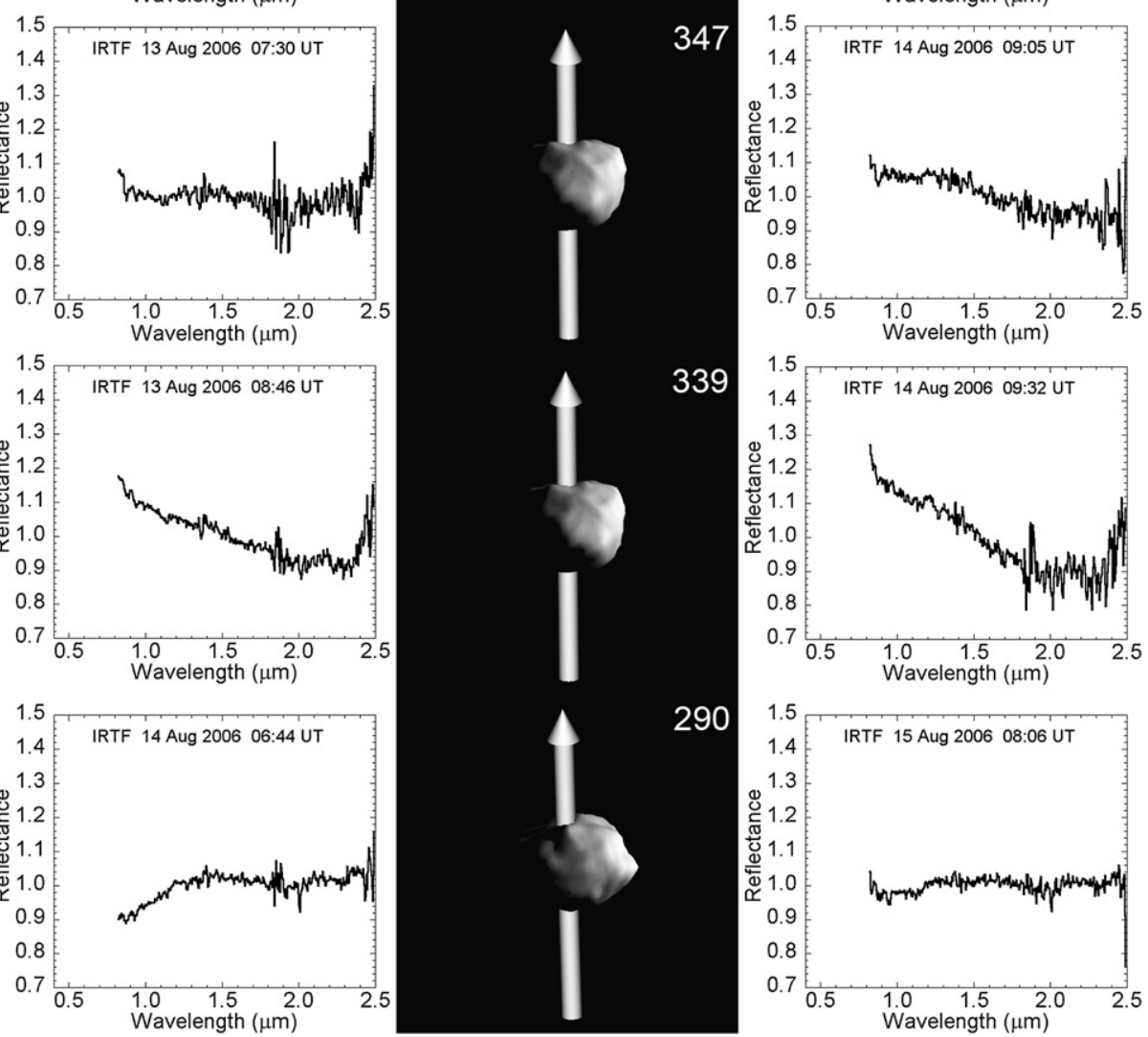

290

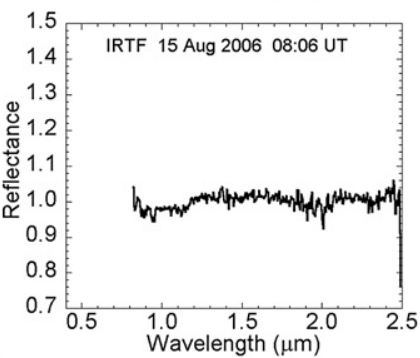

Fig. 15. IRTF spectra with plane-of-sky view of Ra-Shalom at the time spectra were acquired. Dates and times are noted on the spectra. Sub-observer longitudes in degrees are shown in upper right corner of image. All spectra are normalized to 1.0 at $1 \mu \mathrm{m}$.

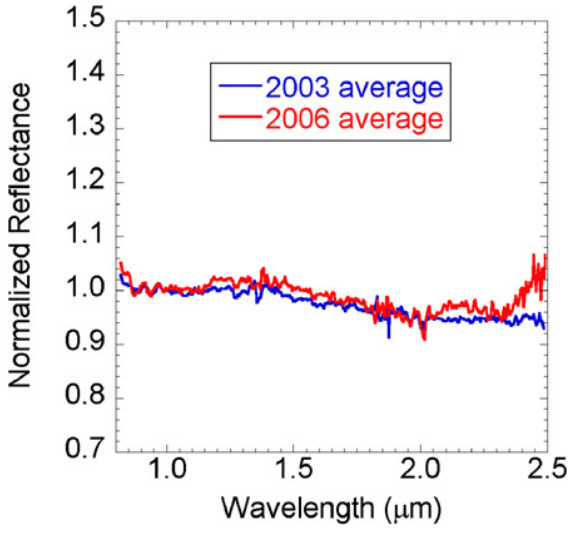

Fig. 16. Comparison of average IR spectra acquired in 2003 and 2006. Spectra normalized to 1.0 at $1 \mu \mathrm{m}$.

tra into three groups for comparative purposes: flat, blue, and red up to $1.3 \mu \mathrm{m}$ and flat thereafter. The data points indicate the sub-observer longitude and our estimated uncertainties for

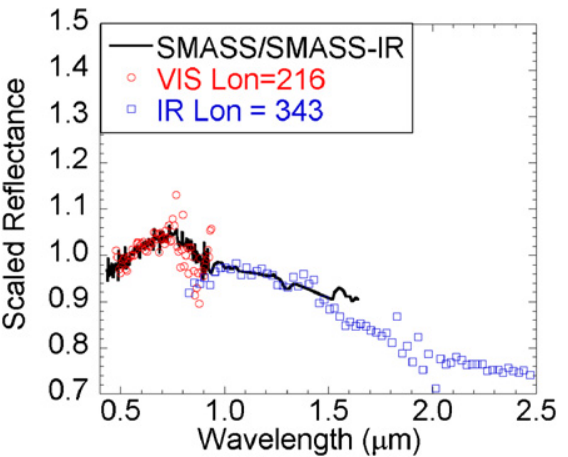

Fig. 17. SMASS and SMASS IR spectra shown superposed with our spectra acquired at similar sub-observer longitudes. See text for acquisition times and estimates of SMASS sub-radar longitudes. Visible spectra are normalized to 1.0 at $0.55 \mu \mathrm{m}$; IR spectra are adjusted vertically to fit.

each point. Uncertainties for the 2003 data are centered on the sub-observer longitude, while those for the 2006 data are onesided and extend to the sub-solar longitude since these are the 


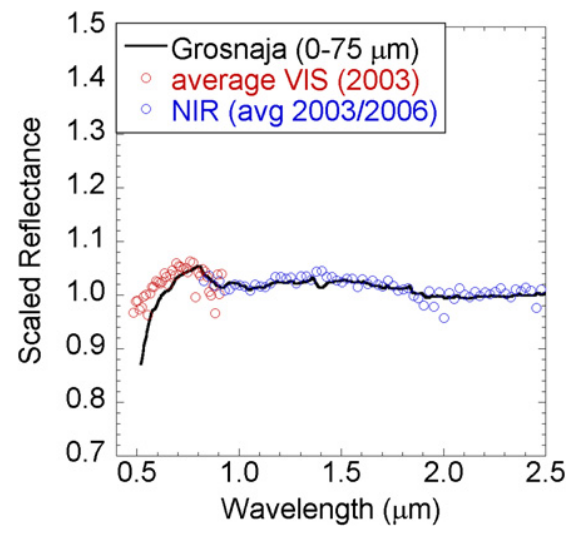

Fig. 18. Sum of 2003 VIS and 2003 and 2006 NIR observations of Ra-Shalom superposed with a laboratory spectrum of fine-grained CV3 Grosnaja (Gaffey, 1976). Telescopic visible spectrum is normalized to 1.0 at $0.55 \mu \mathrm{m}$; NIR and laboratory spectrum are adjusted vertically to fit.

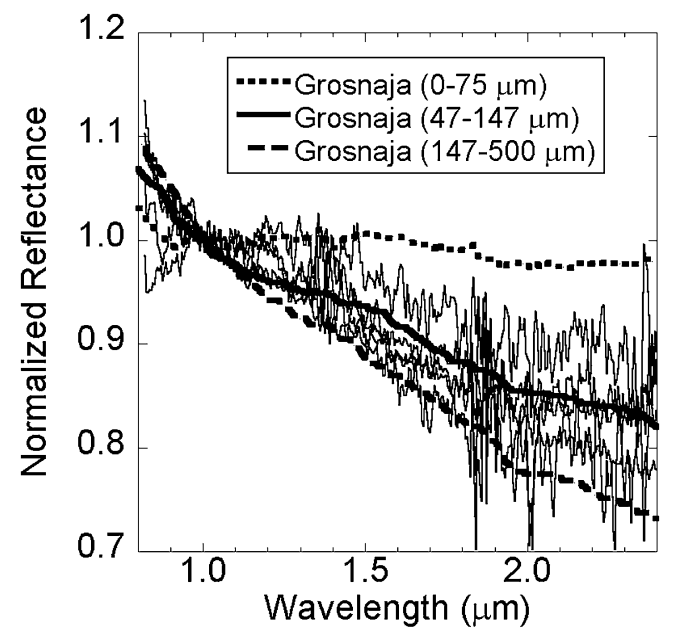

(a)

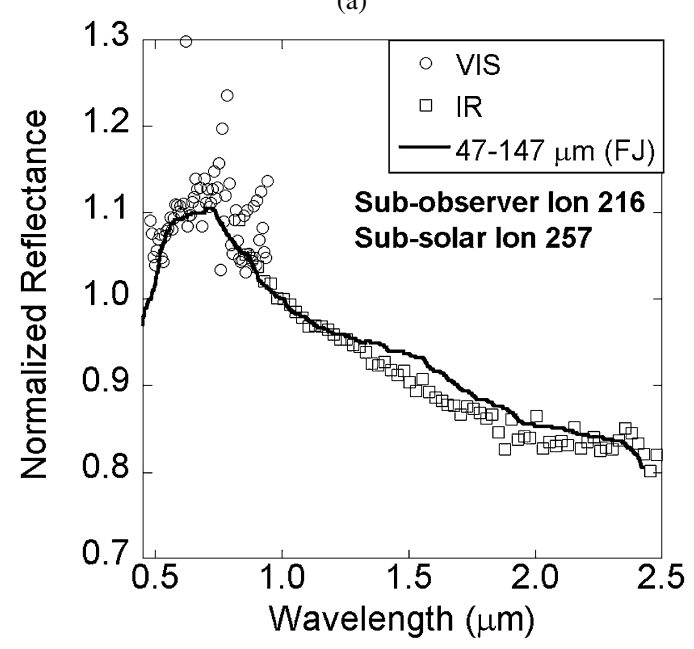

(b)

Fig. 19. (a) IR spectra of Ra-Shalom from 2003 and 2006 (solid, thin) showing a blue slope compared with three different size separates of Grosnaja (bold). All spectra are normalized to a reflectance of 1.0 at $1 \mu \mathrm{m}$. The fine-grain spectrum is from Gaffey (1976) and the coarse-grained spectra are from Johnson and Fanale (1973). (b) Comparison of 2003 spectra at lon $216^{\circ}$ (Fig. 14) with laboratory spectrum of coarse-grain Grosnaja (Johnson and Fanale, 1973). Spectra are normalized to 1.0 at $1 \mu \mathrm{m}$.

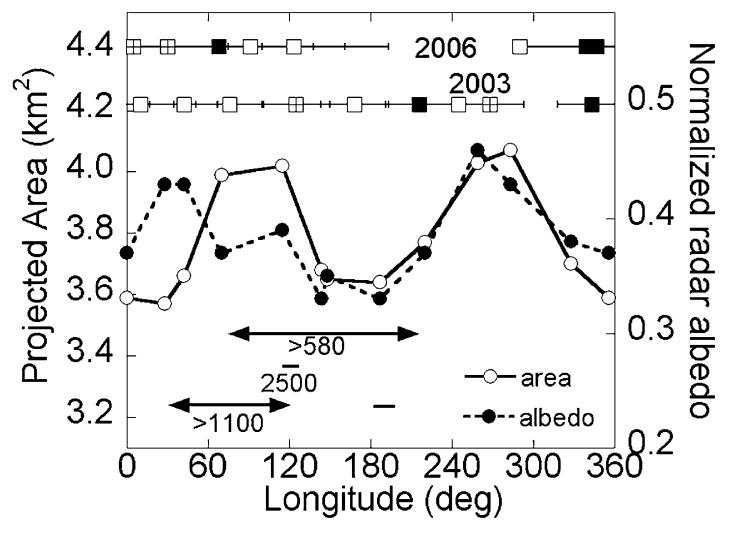

Fig. 20. Plot of Ra-Shalom's projected area and normalized radar albedo (radar cross-section/projected area) versus longitude. At the top are data points showing the sub-observer longitude of our IR spectra (top are 2006 spectra, second row are 2003 spectra); open squares indicate flat spectra, dark squares indicate blue spectra, and squares with a cross indicate spectra that are red up to $1.3 \mu \mathrm{m}$ and flat afterward. The uncertainties for 2003 are shown as $\pm 25^{\circ}$ and for 2006 are one-sided and extend to the sub-solar longitude to include those areas with the highest levels of solar illumination. The arrows and lines at the bottom show the sub-observer longitudes corresponding to the dates and times at which our and previous thermal-infrared data were acquired. We have placed the approximate thermal inertias of each study under the appropriate line ( $>1100$, Harris et al., 1998; 2500, Delbo et al., 2003; > 580, this work and Lim et al., 2005b).

most visibly illuminated regions and, to a first approximation, contribute the most to the observed spectra as discussed earlier (Section 3.2). We also calculate and plot the sub-observer longitudes for our own and for the previous thermal IR data sets.

Comparisons between the 2003 and 2006 rotationally resolved spectra do not show complete agreement. Spectra from both encounters (as well as previous encounters) show similar patterns (e.g., flat, blue, red to flat), suggesting the variations are real. However, we only see agreement at longitudes near $340^{\circ}$ where both encounters exhibit blue spectra. The inconsistencies at other sub-observer longitudes may be due to uncertainties in rotation period (although this should be small) and the different solar phase angles and sub-observer latitudes which, with the additional complication of topography, would emphasize different areas of Ra-Shalom (Figs. 14 and 15).

Given all of our data, the most reasonable interpretation is that Ra-Shalom's near-surface is composed largely of bare or very coarse rock. A thin layer (few $\mathrm{cm}$ or less) of powdered regolith over large fragments (meter-scale) of rock would satisfy the spectral data, the high thermal inertia, and the high radar albedo. Variations in the exposed grain size, which may include sizes ranging from coarse sands to bare rock, can explain much of the spectral variation. The rotational variations in the radar albedo are consistent with regolith that is thicker near the $180^{\circ}$ end and thin, coarse, or absent everywhere else, but especially near lons $30^{\circ}$ and $270^{\circ}$. The high radar albedo near lon $\sim 30^{\circ}$ is likely associated with either the 'knob' or the possible linear features at this end, suggesting a coherent structural feature; there is no obvious structural feature at lon $270^{\circ}$. The blue IR spectra on both dates tend to cluster near the $0^{\circ}$ end, consistent with regolith that is coarse or absent, and are possibly related to the structural knob or linear features. 
A good analogy for the surface texture of Ra-Shalom may be 25143 Itokawa. Recent spacecraft images show large structural elements, boulder fields, and contrasting regions of fines (Fujiwara et al., 2006). Its circular polarization, $\mu_{\mathrm{c}}=$ $0.26 \pm 0.04$, is the same as Ra-Shalom's (Ostro et al., 2005) and it is notable for having a relatively high thermal inertia, estimated to be $10^{2}-10^{3} \mathrm{~J} \mathrm{~m}^{-2} \mathrm{~s}^{-0.5} \mathrm{~K}^{-1}$, comparable to our and other estimates for Ra-Shalom (Yano et al., 2006).

\section{Future opportunities}

Ra-Shalom's next radar encounters occur in August 2009 and September 2013 at modest SNRs of 50/day and 70/day, respectively. Better radar encounters occur in 2016, 2019, 2022, and 2025 when SNRs are 200/day, 500/day, 750/day, and 800/day, respectively. The 2013 and 2016 encounters should be at high sub-radar latitudes and thus well placed for further constraining the pole. The next encounter suitable for spectral measurement at the IRTF (magnitude <15.5) does not occur until October 2016 when Ra-Shalom will be relatively bright (magnitude 14.0) and will present an equatorial aspect suitable for observing rotational variations.

\section{Acknowledgments}

M.K.S. and B.E.C. gratefully acknowledge support from NSF grant AST-0307092. S.L. and F.V. are visiting astronomers at McDonald Observatory of the University of Texas, and this paper includes data taken there. M.K.S. and B.E.C. are visiting astronomers, Infrared Telescope Facility, which is operated by the University of Hawaii under Cooperative Agreement No. NCC 5-538 with the National Aeronautics and Space Administration, Office of Space Science, Planetary Astronomy Program. The Arecibo Observatory is part of the National Astronomy and Ionosphere Center, which is operated by Cornell University under a cooperative agreement with the National Science Foundation. We thank the technical staff at each observatory for help with observations. Some of this work was performed at the Jet Propulsion Laboratory, California Institute of Technology, under contract with the National Aeronautics and Space Administration. This material is based in part upon work supported by the National Aeronautics and Space Administration (NASA) under the Science Mission Directorate Research and Analysis Programs. C.M. was partially supported by NSF grant AST-0205975. D.J.S. acknowledges support from a grant from the Planetary Geology and Geophysics program in NASA's Office of Space Science.

\section{References}

Bell, J.F., 1988. A probable asteroidal parent body for the CV or CO chondrites. Meteoritics 23, 256-257.

Binzel, R.P., Rivkin, A.S., Stuart, J.S., Harris, A.W., Bus, S.J., Burbine, T.H., 2004. Observed spectral properties of near-Earth objects: Results for population distribution, source regions, and space weathering. Icarus 170, 259 294.

Britt, D.T., Consolmagno, G.J., 2003. Stony meteorite porosities and densities: A review of the data through 2001. Meteorit. Planet. Sci. 38, 1161-1180.
Britt, D.T., Yeomans, D., Housen, K., Consolmagno, G., 2002. Asteroid density, porosity, and structure. In: Bottke Jr., W.F., Cellino, A., Paolicchi, P., Binzel, R.P. (Eds.), Asteroids III. Univ. of Arizona Press, Tucson, pp. 485-500.

Burbine, T.H., Binzel, R.P., Bus, S.J., Clark, B.E., 2001. K asteroids and CO3/CV3 chondrites. Meteorit. Planet. Sci. 36, 245-253.

Bus, S.J., Binzel, R.P., 2002a. Phase II of the Small Main-Belt Asteroid Spectroscopic Survey: The observations. Icarus 158, 106-145.

Bus, S.J., Binzel, R.P., 2002b. Phase II of the Small Main-Belt Asteroid Spectroscopic Survey: A feature-based taxonomy. Icarus 158, 146-177.

Carrier III, W.D., Olhoeft, G.R., Mendell, W., 1991. Physical properties of the lunar surface. In: Heiken, G.W., Vaniman, D.T., French, B.M. (Eds.), Lunar Sourcebook: A User's Guide to the Moon. Cambridge Univ. Press, New York, pp. 475-594.

Cushing, M.C., Vacca, W.D., Rayner, J.T., 2004. Spextool: A spectral extraction package for SpeX, a 0.8-5.5 micron cross-dispersed spectrograph. Publ. Astron. Soc. Pacific 116, 362-376.

Delbo, M., Harris, A.W., Binzel, R.P., Pravec, P., Davies, J.K., 2003. Keck observations of near-Earth asteroids in the thermal-infrared. Icarus 166, $116-130$.

Emery, J.P., Sprague, A.L., Witteborn, F.C., Colwell, J.E., Kozlowski, R.W.H., Wooden, D.H., 1998. Mercury: Thermal modeling and mid-infrared (5-12 $\mathrm{mm})$ observations. Icarus 136, 104-123.

Fujiwara, A., and 21 colleagues, 2006. The rubble-pile Asteroid Itokawa as observed by Hayabusa. Science 312, 1330-1334.

Gaffey, M.J., 1976. Spectral reflectance characteristics of the meteorite classes. J. Geophys. Res. 81, 905-920.

Guibout, V., Scheeres, D.J., 2003. Stability of surface motion on a rotating ellipsoid. Celest. Mech. Dynam. Astron. 87, 263-290.

Harris, A.W., 1998. A thermal model for near-Earth asteroids. Icarus 131, 291301

Harris, A.W., Lagerros, J.S.V., 2002. Asteroids in the thermal-infrared. In: Bottke Jr., W.F., Cellino, A., Paolicchi, P., Binzel, R.P. (Eds.), Asteroids III. Univ. of Arizona Press, Tucson, pp. 205-218.

Harris, A.W., Davies, J.K., Green, S.F., 1998. Thermal-infrared spectrophotometry of the near-Earth Asteroids 2100 Ra-Shalom and 1991 EE. Icarus 135, 441-450.

Helin, E.F., Shoemaker, E.M., Wolfe, R.F., 1978. Ra-Shalom: Third member of the Aten class of Earth-crossing asteroids. Bull. Am. Astron. Soc. 10, 732.

Hudson, S., 1993. Three-dimensional reconstruction of asteroids from radar observations. Remote Sensing Rev. 8, 195-203.

Johnson, T.V., Fanale, F.P., 1973. Optical properties of carbonaceous chondrites and their relationship to asteroids. J. Geophys. Res. 78, 8507-8518.

Kaasalainen, M., and 21 colleagues, 2004. Photometry and models of eight near-Earth asteroids. Icarus 167, 178-196.

Lebofsky, L.A., Spencer, J.R., 1989. Radiometry and thermal modeling of asteroids. In: Binzel, R.P., Gehrels, T., Matthews, M.S. (Eds.), Asteroids II. Univ. of Arizona Press, Tucson, pp. 128-147.

Lebofsky, L.A., Lebofsky, M.J., Rieke, G.H., 1979. Radiometry and surface properties of Apollo, Amor, and Aten asteroids. Astron. J. 84, 885-888.

Lebofsky, L.A., Sykes, M.V., Tedesco, E.F., Veeder, G.J., Matson, D.L., Brown, R.H., Gradie, J.C., Feierberg, M.A., Rudy, R.J., 1986. A refined "standard" thermal model for asteroids based on observations of 1 Ceres and 2 Pallas. Icarus 68, 239-251.

Lim, L.F., McConnochie, T.H., Bell, J.F., Hayward, T.L., 2005a. Thermalinfrared $(8-13 \mathrm{~mm})$ spectra of 29 asteroids: The Cornell mid-infrared asteroid spectroscopy (MIDAS) survey. Icarus 173, 385-408.

Lim, L.F., Emery, J.P., McConnochie, T.H., 2005b. Thermal-infrared (8-13 micron) spectra of the NEA 2100 Ra-Shalom. Bull. Am. Astron. Soc. 37, 629 (abstract).

Lord, S., 1992. A New Software Tool for Computing Earth's Atmospheric Transmission of Near- and Far-Infrared Radiation. NASA Technical Memorandum 103957.

Magri, C., Consolmagno, G.J., Ostro, S.J., Benner, L.A.M., Beeney, B.R., 2001. Radar constraints on asteroid regolith properties using 433 Eros as ground truth. Meteorit. Planet. Sci. 36, 1697-1709.

Magri, C., Nolan, M.C., Ostro, S.J., Giorgini, J.D., 2007a. A radar survey of main-belt asteroids: Arecibo observations of 55 objects during 1999-2003. Icarus 186, 152-177. 
Magri, C., Ostro, S.J., Scheeres, D.J., Nolan, M.C., Benner, L.A.M., Margot, J.L., 2007b. Radar observations and a physical model of Asteroid 1580 Betulia. Icarus 186, 152-177.

McFadden, L.A., Gaffey, M.J., McCord, T.B., 1984. Mineralogical-petrological characterization of near-Earth asteroids. Icarus 59, 25-40.

Mitchell, D.L., Ostro, S.J., Hudson, R.S., Rosema, K.D., Campbell, D.B., Velez, R., Chandler, J.F., Shapiro, I.I., Giorgini, J.D., Yeomans, D.K., 1996. Radar observations of Asteroids 1 Ceres, 2 Pallas, and 4 Vesta. Icarus 124, 113-133.

Ostro, S.J., Harris, A.W., Campbell, D.B., Shapiro, I.I., Young, J.W., 1984. Radar and photoelectric observations of Asteroid 2100 Ra-Shalom. Icarus 60, 391-403.

Ostro, S.J., Hudson, R.S., Nolan, M.C., Margot, J.L., Scheeres, D.J., Campbell, D.B., Magri, C., Giorgini, J.D., Yeomans, D.K., 2000. Radar observations of Asteroid 216 Kleopatra. Science 288, 836-839.

Ostro, S.J., Hudson, R.S., Benner, L.A.M., Giorgini, J.D., Magri, C., Margot, J.L., Nolan, M.C., 2002. Asteroid radar astronomy. In: Bottke Jr., W.F., Cellino, A., Paolicchi, P., Binzel, R.P. (Eds.), Asteroids III. Univ. of Arizona Press, Tucson, pp. 151-169.

Ostro, S.J., Benner, L.A.M., Nolan, M.C., Magri, C., Giorgini, J.D., Scheeres, D.J., Broschart, S.B., Kaasalainen, M., Vokrouhlicky, D., Chesley, S.R., Margot, J.L., Jurgens, R.F., Rose, R., Yeomans, D.K., Suzuki, S., DeJong, E.M., 2005. Radar observations of Asteroid 25143 Itokawa (1998 SF36). Meteorit. Planet. Sci. 39, 407-424.

Pravec, P., Harris, A.W., 2007. Binary asteroid population. 1. Angular momentum content. Icarus 190, 250-259.
Pravec, P., Wolf, M., Sarounova, L., 1998. Lightcurves of 26 near-Earth asteroids. Icarus 136, 124-153.

Prockter, L., Thomas, P., Robinson, M., Joseph, J., Milne, A., Bussey, B., Veverka, J., Cheng, A., 2002. Surface expressions of structural features on Eros. Icarus $155,75-93$.

Rayner, J.T., Toomey, D., Onaka, P., Denault, A., Stahlberger, W., Vacca, W., Cushing, M., 2003. SpeX: A medium-resolution 0.8-5.5 micron spectrograph and imager for the NASA Infrared Telescope Facility. Publ. Astron. Soc. Pacific 115 (805), 362-382.

Scheeres, D.J., Ostro, S.J., Hudson, R.S., Werner, R.A., 1996. Orbits close to Asteroid 4769 Castalia. Icarus 121, 67-87.

Sears, D.W.G., and 19 colleagues, 2001. Near-Earth asteroid sample return missions. In: Am. Inst. Aeron. Astron. Space 2001, AIAA-2001-4728 (abstract).

Shepard, M.K., Benner, L.A.M., Ostro, S.J., Harris, A.W., Rosema, K.D., Shapiro, I.I., Chandler, J.F., Campbell, D.B., 2000. Radar observations of Asteroid 2100 Ra-Shalom. Icarus 147, 520-529.

Veeder, G.J., Hanner, M.S., Matson, D.L., Tedesco, E.F., Lebofsky, L.A., Tokunaga, A.T., 1989. Radiometry of near-Earth asteroids. Astron. J. 97, 12111219.

Vilas, F., Smith, B.A., 1985. Reflectance spectrophotometry $(\sim 0.5-1.0 \mu \mathrm{m})$ of outer-belt asteroids: Implications for primitive, organic Solar System material. Icarus 64, 503-516.

Vokrouhlicky, D., Capek, D., Chesley, S.R., Ostro, S.J., 2005. Yarkovsky detection opportunities. I. Solitary asteroids. Icarus 173, 166-184.

Yano, H., and 19 colleagues, 2006. Touchdown of the Hayabusa spacecraft at the Muses Sea on Itokawa. Science 312, 1350-1353. 